\title{
Cancer avatars derived from genetically engineered pluripotent stem cells allow for longitudinal assessment of tumor development.
}

\section{Authors:}

Tomoyuki Koga ${ }^{1,2^{*}}$, Isaac A. Chaim ${ }^{3 *}$, Jorge A. Benitez ${ }^{1 \dagger}$, Sebastian Markmiller ${ }^{3 \dagger}$, Alison D. Parisian $^{1,4}$, Robert F. Hevner ${ }^{4}$, Kristen M. Turner ${ }^{1}$, Florian M. Hessenauer ${ }^{1}$, Matteo D’Antonio ${ }^{5}$, Nam-phuong D. Nguyen ${ }^{6}$, Shahram Saberi ${ }^{7}$, Jianhui Ma ${ }^{1}$, Shunichiro Miki ${ }^{1}$, Antonia D. Boyer ${ }^{1}$, John Ravits ${ }^{7}$, Kelly A. Frazer ${ }^{5,8}$, Vineet Bafna ${ }^{6}$, Clark C. Chen ${ }^{2}$, Paul S. Mischel ${ }^{1,4}$, Gene W. Yeo $^{3,5 \S}$, and Frank B. Furnari ${ }^{1,4 \S}$.

\section{Affiliations:}

${ }^{1}$ Ludwig Cancer Research, San Diego Branch, La Jolla, CA 92093, USA.

${ }^{2}$ Department of Neurosurgery, University of Minnesota, Minneapolis, MN 55455, USA.

${ }^{3}$ Department of Cellular and Molecular Medicine, University of California at San Diego, La Jolla, CA 92093, USA.

${ }^{4}$ Department of Pathology, University of California at San Diego, La Jolla, CA 92093, USA.

${ }^{5}$ Institute for Genomic Medicine, University of California at San Diego, La Jolla, CA 92093, USA.

${ }^{6}$ Department of Computer Science and Engineering, University of California at San Diego, La Jolla, CA 92093, USA.

${ }^{7}$ Department of Neuroscience, University of California at San Diego, La Jolla, CA 92093, USA.

${ }^{8}$ Department of Pediatrics and Rady Children's Hospital, University of California at San Diego, La Jolla, CA 92093, USA. 
*These authors contributed equally to this work.

${ }^{\dagger}$ These authors contributed equally to this work.

${ }^{\S}$ Correspondence to: Frank B. Furnari, Ludwig Cancer Research, University of California at San

Diego, 9500 Gilman Dr., CMM-East Room 3055, La Jolla, CA, 92093-0660. Phone: 858-534-

7819. Fax: 858-534-7750. Email: ffurnari@ucsd.edu.

Gene W. Yeo, Dept of Cellular and Molecular Medicine, University of California at San Diego, 2880 Torrey Pines Scenic Drive, La Jolla, CA 92037. Phone: 858534 9321. Email:

geneyeo@ucsd.edu 


\begin{abstract}
Many current cellular models aimed at elucidating cancer biology do not recapitulate pathobiology including tumor heterogeneity, an inherent feature of cancer that underlies treatment resistance. Here we introduce a new cancer modeling paradigm using genetically engineered human pluripotent stem cells (hiPSCs) that capture authentic cancer pathobiology. Orthotopic engraftment of neural progenitor cells derived from hiPSCs that have been genomeedited to contain tumor-associated genetic driver mutations revealed by The Cancer Genome Atlas project for glioblastoma (GBM) result in formation of high-grade gliomas. As observed in GBM patient samples, these models harbor inter-tumor heterogeneity resembling different GBM molecular subtypes, and intra-tumor heterogeneity. Further, re-engraftment of primary tumor neurospheres generates secondary tumors with features characteristic of patient samples and present mutation-dependent patterns of tumor evolution. Thus, these cancer avatar models provide a platform for a comprehensive longitudinal assessment of human tumor development as governed by molecular subtype mutations and lineage-restricted differentiation.
\end{abstract}

\title{
INTRODUCTION
}

Effective modeling of cancer has been a conceptual cornerstone in the field of oncology for studying pathobiology and identifying therapeutic targets. In the case of glioblastoma (GBM), the most common primary malignant tumor of the central nervous system ${ }^{1}$, mouse models of GBM-like tumors generated through the genetic disruption of different combinations of core tumor suppressors and/or by introduction of oncogenes such as $S r c, K$-ras, $H$-ras, PDGFB, and $E G F R v I I^{2}$ are available to investigate the biology of these aggressive tumors or to test possible treatments in preclinical settings ${ }^{3}$. These mouse models are suitable to investigate pathology of 
genetically defined gliomas and useful for drug testing, but typically lack the intra-tumor heterogeneity that is observed in human gliomas ${ }^{4}$. Additionally, while human astrocytes engineered with combinations of human TERT and $H$-Ras expression and inhibition of the TP53 pathway either by SV40 T/t-Ag or by HPV E6 and E7 generate gliomas with high-grade histology ${ }^{5,6}$, how well these models recapitulate the full spectrum of glioma pathobiology, especially in terms of GBM heterogeneity, has not been well defined. In contrast, patient derived xenografts (PDX) have been useful to study inter- and intra-tumoral heterogeneity ${ }^{7,8}$ and sensitivity to pathway-specific therapies ${ }^{9}$, however they do not allow for experimental standardization or afford analysis of the effects of molecular subtype mutations on tumor evolution.

The progress in human stem cell technologies and genome editing using site-specific nucleases such as ZFN, TALEN, and CRISPR/Cas9 has broadened the field of human disease modeling ${ }^{10}$. This combination of human stem cell and genome editing promises great potential when applied to cancer models. The first such model generated utilized colon organoids derived from human intestinal crypt stem cells engineered with four or five mutations common in colorectal cancers $^{11,12}$. Later, a brain tumor model deleted for PTEN by TALEN-mediated homologous recombination (HR) led to the reprograming of human neural stem cells towards a cancer stem cell-like phenotype ${ }^{13}$. However, it remains unknown if these cancer models generated through genome editing harbor authentic pathological features of cancers, including tumor heterogeneity and clonal evolution.

Here, we established a robust platform in an isogenic background, which uses CRISPR/Cas9 genome editing technology and serial in vivo engraftments enabling longitudinal assessment of human high-grade glioma (HGG) models containing combinations of genetic 
alterations observed in proneural and mesenchymal GBM molecular subtypes. We further investigated how closely these models recapitulate pathobiology of the disease and evaluate their utility as an avatar platform for future studies on tumor biology and evolution.

\section{RESULTS}

\section{Neural progenitor cells engineered with GBM-associated mutations give rise to brain tumors resembling HGG.}

We first introduced two different combinations of driver mutations into human induced pluripotent stem cells (iPSCs) by CRISPR/Cas9 genome editing ${ }^{14,15}$ (Fig. 1a,b). One combination of deletions targeted tumor suppressor genes PTEN and NF1, which are commonly altered together in the mesenchymal subtype of $\mathrm{GBM}^{16,17}$. A second combination of deletions targeted TP53 and exons 8 and 9 of PDGFRA (PDGFRA $48-9)$. This creates a constitutively active truncating PDGFRA mutation observed in $40 \%$ of $P D G F R A$ amplified $\mathrm{GBM}^{18}$, resulting in a genotype commonly found in the proneural subtype of isocitrate dehydrogenase-wildtype $\mathrm{GBM}^{16,17}$. The genetic modifications in single clones were confirmed by genotyping PCR (Fig. 1c) and RT-qPCR (Fig. 1d). Edited iPSC clones with desired mutations were differentiated into neural progenitor cells (NPCs), using a small molecule protocol ${ }^{19}$ and differentiation status was confirmed by downregulation of pluripotency markers, Nanog and Oct4, and corresponding upregulation of NPC markers, Pax6, Nestin, and Sox1 (Fig. 1e).

We next evaluated if these genetically modified NPCs were capable of forming orthotopic tumors in immunocompromised mice (Fig. 1a). When edited NPCs were engrafted in the brains of four Nod/Scid mice, $P T E N^{/-} ; N F 1^{-/-} \mathrm{NPCs}$ and $T P 53^{-/} ; P D G F R A^{48-9}$ NPCs each formed brain tumors with median survival of 141, and 119.5 days, respectively (Fig. 1f) . 
Pathological assessment of $P T E N^{-/} ; N F 1^{-/}$tumors revealed regions of hypercellularity with occasional mitoses (Fig. 2a), and in one out of four tumors there was biphasic dense glial and loose mesenchymal/sarcoma morphologies, typical of gliosarcoma (Fig. 2b). Additionally, regions of necrosis (Fig. 2c), vascular endothelial proliferation (Fig. 2d), subarachnoid spread (Fig. 2e), perineuronal satellitosis, and subpial accumulation of tumor cells (Fig. 2f), were also apparent. The tumors were consistently positive for GFAP $(3+$ in $6 / 6$ high power fields) (Fig. $2 \mathrm{~g}$, Supplementary Fig. 1a) and Olig 2 (3+ in 4/6 high power fields, 2+ in 2/6 high power fields) (Fig. 2h, Supplementary Fig. 1b), and highly proliferative as indicated by Ki-67 staining $\left(35.44 \pm 1.435 \%\right.$; mean \pm SEM) (Fig. 2i, Supplementary Fig. 1c). TP53 ${ }^{-/-}$;PDGFRA ${ }^{48-9}$ tumors presented nodular growth of a primitive neuronal component (dark purple) intermingled with glial components (Fig. 2j), rosettes with neuropil-like texture (Fig. 2k), a serpiginous zone of pseudopalisading necrosis (Fig. 21), and intraventricular growth (Fig. 2m). These tumors were positive for GFAP (3+ in 6/6) (Fig. 2n, Supplementary Fig. 1a) and Olig 2 (3+ in 6/6) (Fig. 2o, Supplementary Fig. 1b), and also highly positive for Ki-67 staining (27.79 $\pm 5.731 \%$; mean \pm SEM) (Fig. 2p, Supplementary Fig. 1c). In terms of WHO grade, three and one out of four tumors were scored as grade 4 and grade 3 , respectively for $P T E N^{{ }^{-}} ; N F 1^{-/-}$tumors, and four out of four tumors were scored as grade 4 for $T P 53^{-/} ; P D G F R A^{48-9}$ tumors (Supplementary Fig. 2). In contrast, $P T E N^{-/}$and $T P 53^{-/}$singly edited NPCs did not form tumors in the brain over the same time span (Fig. 1f, Supplementary Fig. 3a,b), while unedited iPSCs formed teratoma-like tumors (Supplementary Fig. 4). Lack of teratomas after NPC injection suggests a high efficiency of differentiation to NPCs. These results illustrate that using this modeling paradigm, small numbers of known driver mutations found in GBM are sufficient for phenotypic recapitulation of human HGG tumors. 


\section{Cells from iHGG models can be cultured in vitro and re-engrafted to form secondary \\ tumors.}

One of the benefits of using PDX models in cancer research is that they can be cultured in vitro and be re-engrafted in animals, thus enabling both in vitro and in vivo analyses ${ }^{20}$. We evaluated if our induced HGG (iHGG) models could be used in a similar manner. Dissociated tumors obtained from the mouse brains were sorted for human cells using a human MHC antibody, followed by propagation of isolated cells in the same neurosphere conditions used for GBM PDX spheres $^{21}$, which confirmed iHGG sphere formation capability (Fig. 3a). These iHGG spheres possessed the same genotypes as the corresponding input NPCs (Supplementary Fig. 5). Extreme limiting dilution assays ${ }^{22}$ showed that iHGG spheres had greater self-renewal capacity, a feature of cancer stem cells, when compared to pre-engraftment NPCs (Fig. 3b), again highlighting gain of cancerous phenotypes of iHGG cells compared to original input cells. We then evaluated if these iHGG-derived sphere cells maintained tumorigenic capacity by secondary orthotopic engraftment (Fig. 3c). When injected in the brains of Nod/Scid mice, $\mathrm{PTEN}^{/-} ; \mathrm{NF}^{-/}$and $T P 53^{-/} ; P D G F R A^{48-9} \mathrm{iHGG-derived} \mathrm{sphere} \mathrm{cells} \mathrm{formed} \mathrm{tumors} \mathrm{with} \mathrm{a}$ shortened latency period of median 76.5 days and 34.5 days, respectively $(\mathrm{p}=0.0005)$ (Supplementary Fig. 6). We also tested if these models can be used for in vivo drug treatment experiments comparable to those applied to PDX lines by treating orthotopically engrafted animals with temozolomide (TMZ), a DNA-alkylating chemotherapeutic agent used for standard care treatment of GBM patients ${ }^{23} . T^{2} 53^{-/-} ; P D G F R A^{48-9}$ iHGGs proved to be more sensitive to TMZ compared to $P T E N^{--} ; N F 1^{-/}$iHGGs (Fig. 3d). PTEN ${ }^{--} ; N F 1^{-/}$iHGGs were found to express higher levels of $O^{6}$-methylguanine DNA methyl transferase (MGMT) (Fig. 3e), which is 
associated with resistance to TMZ in GBM patients ${ }^{24}$, compared to TP53 $^{-/-} ;$PDGFRA ${ }^{48-9}$ iHGGs. An alternative explanation of this differential sensitivity, through MGMT-independent mechanisms in the context of TP53 alteration ${ }^{25,26}$ cannot be eliminated.

\section{iHGGs recapitulate molecular and genetic hallmarks of patient tumors.}

We further investigated if these iHGGs showed inter- and intra-tumor heterogeneity, which is another hallmark of GBM and cancer in general $^{27}$. This important feature of cancer has not been well studied in previous models to date. To investigate the robustness of our iHGG models we performed in triplicate, single-cell RNA sequencing (scRNA-seq) using primary iHGG spheres, secondary iHGG tumor cells obtained from orthotopic injection of the primary spheres, as well as secondary spheres derived by in vitro culture of the secondary tumor cells for both genotypes, for a total of 14 samples (Fig. 4a).

Visual analysis of all the samples by Uniform Manifold Approximation and Projection (UMAP) reveals clear structural stratification between primary and secondary spheres of the same genotype as well as between spheres and tumors (Fig. 4b). However, the greatest variation appears between the two iHGG models of different genotypes. This inter-tumor heterogeneity between iHGG models was not apparent in pre-engraftment NPCs with different gene edits (Supplementary Fig. 7). In fact, the strongest driver of transcriptomic differences is genotype, as shown by the clear split between them, regardless of their origin (spheres or tumors, primary or secondary) for the strongest Principal Component (PC), pc1 (Fig. 4c). These findings continue to support the notion that a small number of driver mutations are sufficient for the development of such pathognomonic inter-tumor heterogeneity that arises through the process of transformation. 
Given that our models were engineered to recapitulate different clinical GBM molecular subtypes, specifically proneural and mesenchymal, we sought to determine if our samples manifested transcriptomic GBM signatures as established previously ${ }^{8,16} \cdot$ TP53 $^{-/-} ; P D G F R A^{48-9}$ iHGG show upregulation of genes characteristic of the proneural subtype, while the $P T E N^{\prime-}$ ;NF1 $1^{-/}$iHGG show a mesenchymal subtype signature for both spheres and tumors (Fig. 4d, and Supplementary Fig. 8). Subtype scores involving all expressed genes under each subtype show similar trends, with higher proneural scores for $T P 53^{-/} ; P D G F R A^{48-9} \mathrm{iHGG}$ and higher mesenchymal scores for $P T E N^{-\alpha} ; N F 1^{-/}$iHGG (Supplementary Fig. 9). Interestingly, TP53 ${ }^{-/-}$ ;PDGFRA ${ }^{48-9}$ iHGG samples also show increased classical subtype scores. Importantly, when examined at single cell resolution, each sample shows intra-tumor heterogeneity with different populations of cells presenting signatures of different subtypes (Supplementary Fig. 9), as is characteristic of GBM patient samples ${ }^{27}$. Additionally, all samples are comprised of populations of cycling and non-cycling cells (Supplementary Fig. 10a,b), which is also characteristic of patient samples and is in juxtaposition with other in vitro GBM models, where almost $100 \%$ of cells are cycling ${ }^{27}$. Finally, in agreement with previous literature on patient samples, cells with high proneural scores also score highly on stemness as is the case for our $T P 53^{-/-} ; P D G F R A^{48-9}$ iHGG samples (Supplementary Fig. 10c-e) ${ }^{27}$. In conclusion, our results highlight the robustness of our iHGG models, for both spheres and tumors, in recapitulating hallmarks of patient GBM samples as is the case for cellular inter- and intra-tumor heterogeneity, subtype signatures and cycling and stemness scores.

\section{Genetically distinct iHGG models present different patterns of longitudinal evolution.}


It is also apparent from the UMAP plots that the transcriptomic signature of primary spheres evolves as they are passaged through mice, excised, and cultured in vitro. In fact, by analyzing separately the transition of each genotype model from primary to secondary spheres, we gain insights into the biology of the tumors as well as the role that in vivo passaging plays. We performed unsupervised Louvain clustering of $\mathrm{PTEN}^{-/} ; \mathrm{NF}^{-/-}$iHGG primary and secondary

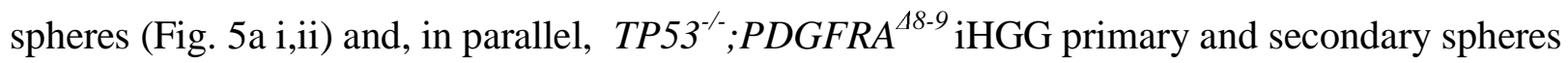
(Fig. $5 \mathrm{~b}$ i,ii) and found 8 and 15 distinct clusters, respectively. In both cases primary spheres are represented, almost exclusively, by unique clusters not found in any secondary spheres. Remarkably, all three $P T E N^{--} ; N F 1^{-/}$iHGG secondary spheres are found in very similar proportions in the remaining clusters (Fig. 5a iii,iv). In stark contrast, each of the TP53 ${ }^{-/-}$ ;PDGFRA ${ }^{48-9}$ iHGG secondary spheres show unique cluster makeups (Fig. 5b iii,iv).

Moreover, when the differentially expressed genes of each cluster are subjected to gene ontology (GO) analysis, different patterns emerge for each iHGG model. $P T E N^{\prime-} ; N F 1^{-/}$iHGG spheres are subdivided in two broad categories, namely cell cycle or cell motility and extra- and intra-cellular fiber reorganization, with several cluster sharing a variety of GO terms (Fig. 5c). In fact, both categories appear to be exacerbated following tumor formation in mice as shown by the increase in the number of GO terms associated with each secondary sphere cluster. This increase in cell motility terms supports our observation regarding more prominent diffuse invasion of $P T E N^{/-} ; N F 1^{-/}$iHGG compared with $T P 53^{-/-} ; P D G F R A^{48-9}$ iHGG (Supplementary Fig. 11). In contrast, in addition to cell cycle associated GO terms, $T P 53^{-/} ; P D G F R A^{48-9}$ iHGG spheres are represented by diverse GO terms, many of which are unique to each secondary sphere, including glial cell differentiation, response to hypoxia, embryonic morphogenesis and, similarly to $P T E N^{/-} ; \mathrm{NF}^{-/}$iHGG spheres, cell motility associated terms (Fig. 5d). Likewise, the 
mesenchymal signature of $P T E N^{-\alpha} ; N F 1^{-/}$iHGG spheres is homogenous across most clusters whereas proneural scores are heterogeneous in nature (Supplementary Fig. 12,13). Overall, even though both iHGG models show transcriptional drift from primary to secondary spheres, $T P 53^{-/-}$ ;PDGFRA ${ }^{48-9}$ iHGGs appear to show a less unidirectional path with increased heterogeneity.

We previously reported that extrachromosomal DNA (ecDNA) is prevalent in many cancer types, especially in GBM, and that ecDNA is associated with resistance to drug treatment and rapid evolution of tumor heterogeneity ${ }^{28,29}$. To determine if our iGBM models recapitulated the generation of ecDNA, we first investigated if the original input NPCs possessed karyotype abnormalities or traces of ecDNA. Based on DAPI staining of metaphase spreads and digital karyotyping, $P T E N^{--} ; \mathrm{NF}^{-/}$iHGG cells were karyotypically normal (Fig. 6A). In sharp contrast, metaphase spreads of cells obtained from $T P 53^{-/} ; P D G F R A^{48-9}$ iHGGs showed small DAPIstained dots adjacent to chromosomes, suggestive of ecDNA (Fig. 6B), consistent with our previous findings in GBM tumor samples ${ }^{29}$. Furthermore, double minute-like structures became more apparent in the secondary tumors obtained by re-engraftment of the primary spheres (Fig. 6C), and were replication competent as indicated by incorporation of EdU (Fig. 6D). The TP53 ${ }^{-/}$ ;PDGFRA ${ }^{48-9}$ iHGGs also presented striking numerical and structural chromosome alterations (Fig. 6E). This supports a clonally unstable nature of the $T P 53^{-/} ; P D G F R A^{48-9}$ model, where genomic instability or ecDNA could be driving dynamic accelerated clonal evolution ${ }^{29,30}$.

\section{iHGG tumors confirm features characteristic of patient tumor samples.}

We also applied unsupervised Louvain clustering of $P T E N^{--} ; N F 1^{-/}$iHGG secondary tumors (Fig. 7ai) and, in parallel, TP53 ${ }^{-/} ; P D G F R A^{48-9}$ iHGG secondary tumors (Fig. 7bi) to further compare inter- and intra-tumor variability of our iHGG tumor models and found 7 and 8 distinct 
clusters, respectively (Fig. 7aii, 7bii). Each one of the clusters is represented by a unique set of differentially expressed genes which, if stratified by sample, show no clear patterns, as would be expected when intra-tumor heterogeneity is present at the single cell level (Supplementary Fig. 14). Furthermore, $P T E N^{/-} ; N F 1^{-/}$iHGG secondary tumors appear more homogenous (Fig. 7aiii,iv) than their $T P 53^{-/} ; P D G F R A^{48-9}$ counterparts as each one of the triplicates is represented in all clusters (Fig. 7biii,iv). On the contrary, $T P 53^{-/} ; P D G F R A^{48-9}$ iHGG secondary tumors show clusters containing cells from all samples but also several clusters comprised of only two or almost exclusively one sample, indicating increased inter-tumor variability.

Significantly, when GBM molecular subtypes of each cluster are inspected, $P T E N^{-1} ; N F 1^{-}$ / samples show, for the most part, a homogenous mesenchymal signature, with the exception of two clusters ('E' and 'G') (Fig. 7c). In stark contrast, subtype signatures of each cluster derived from $T P 53^{-/} ; P D G F R A^{48-9}$ tumors show unique combinations, with three clusters accounting for the majority of the proneural signature and smaller contributions by the remaining clusters with the exception of cluster 'O' which has a clear mesenchymal signature (Fig. 7d). Notably, for both models, intra-tumor heterogeneity is also observed as each cluster presents signatures of different molecular subtypes, a hallmark of GBM patient samples ${ }^{27}$ (Supplementary Fig. 12,13). Finally, some $T P 53^{-/} ; P D G F R A^{48-9}$ tumor clusters also score higher for stemness in comparison to $\mathrm{PTEN}^{-/} ; \mathrm{NFI}^{-/}$clusters; while both models show a heterogenous composition of cycling and non-cycling cells (Fig. 7e,f). In conclusion, our isogenic models faithfully recapitulate HGG pathobiology, including inter- and intra-tumor heterogeneity, differential drug sensitivity, extrachromosomal DNA amplifications, and rapid clonal evolution. Variations of this tumor avatar platform can be applied to different types of cancers and will allow, amongst other things, 
the study of clonal evolution, longitudinal assessment in vitro and in vivo, and genotype-based therapeutic vulnerabilities deciphered in an isogenic background.

\section{DISCUSSION}

We generated isogenic iHGG models from hiPSCs by introducing different combinations of genetic alterations characteristic of this disease. These models both recapitulated pathological features of HGG, but at the same time, displayed distinct mutation-dependent variation in histological morphology, gene expression, and ploidy. Some of our findings were consistent with previous mouse models. For example, NF1-deleted tumors and PDGF-driven tumors showed features of mesenchymal and proneural subtype, respectively in mouse models ${ }^{31-33}$, where the former tumors were resistant to $\mathrm{TMZ}$ while the latter tumors were sensitive to the drug $^{31}$. Such consistency among our models, patient samples, and previous models suggests the key roles of small numbers of driver mutations in determining tumors phenotypes. The heterogeneity presented in our models is an essential feature for the proper modeling of GBM as this prominent characteristic is a confounding aspect making these tumors difficult to treat. Although there have been several models generated from pluripotent cells and genome editing technologies to date ${ }^{13}$, our iHGG models are the first human pluripotent cell derived models that present exact pathognomonic features of GBM, and first to reproduce the heterogeneity of the disease from isogenic cells. These approaches have been applied for models of other brain tumors such as medulloblastoma ${ }^{34}$. Furthermore, recent development of $3 \mathrm{D}$ in vitro brain tumor models using cerebral organoid suggests that several different combinations of oncogenic mutations give rise to expanding tumor-like components within organoids, which would approximate in vivo conditions better than conventional cell culture conditions ${ }^{35}$. Such 
applications of advanced differentiation technology of pluripotent stem cells and genome engineering enabling introduction of genetic alterations actually observed in patient samples, could further develop the next generation of cancer models.

A combination of EGFR activation and inactivation of Ink4a/Arf, which are common cooccurring genetic alterations observed in high grade gliomas, have been shown to play a role in dedifferentiation of astrocytes through the process of gliomagenesis ${ }^{36}$. How the genetic alterations we engineered in NPCs contribute to the formation of iHGGs is to be further studied, and presents an ideal platform to investigate mechanisms promoting transformation guided by cell lineage.

Once xenograft tumors were obtained with our proneural and mesenchymal iHGG models, cells from these tumors maintained tumorigenicity and formed secondary tumors in vivo resembling the original tumors, as also seen in PDX models ${ }^{37}$. Owing to this characteristic, the iHGG cells can be passaged and maintained as cell lines once tumors are obtained. Further, as shown here, it is quite feasible to introduce different combinations of genetic alterations in hiPSCs, and such different edits result in divergent phenotypes. Thus, by expanding our geneediting spectrum, we expect that these models would enable us to evaluate the influence of select driver genetic alterations found in different cancer types, which is less feasible with PDX models due to numerous acquired passenger mutations and genetic backgrounds that are highly variable sample to sample.

Another striking finding in our iHGG models was aneuploidy accompanied by ecDNA, observed in $T P 53^{--} ; P D G F R A^{48-9}$ tumors. To best of our knowledge, this model represents the first cancer model derived from hiPSCs showing ecDNA amplification. In our previous analyses, ecDNA was observed in more than $80 \%$ of PDX cell lines derived from $\mathrm{GBM}^{29}$, suggesting that 
ecDNA formation is a fundamental feature in the pathogenesis of GBM. Interestingly, a mouse model with a combination of $C D K N 2 A^{-/}$and a PDGFRA point mutation showed brain tumors with double minute chromosomes or ecDNA ${ }^{38}$, while another $C D K N 2 A^{-/}$mouse model that generated brain tumors after irradiation similarly had ecDNA ${ }^{39}$. Using human intestinal stem cells that were edited for the most commonly mutated colorectal cancer genes (APC, TP53, KRAS and SMAD4), extensive aneuploidy occurred and these quadruple mutant cells grew as tumors in immunocompromised mice with features of invasive carcinoma ${ }^{11}$. Together with our results, these previous models suggest alterations in TP53 or CDKN2A, which are commonly affected in GBM as well as other cancer types, play an essential role in the genesis of chromosomal instability that results in aneuploidy or ecDNA formation.

In summary, we propose a new modeling system for HGG by introducing different combinations of essential genetic alterations in hiPSCs, which result in tumor avatars faithfully recapitulating histology, gene expression signatures, and cytogenetic features of HGG. As these avatars are faithfully expressing gene expression signatures characteristic of GBMs, we expect that these models will be a useful platform to study cancer biology based on genetic drivers, cell of origin defined by the differentiation program of genome-edited iPSCs, and possible other parameters such as xenograft location and gender differences.

\section{ACKNOWLEDGEMENTS}

We thank W.K. Cavenee, A.H. Thorne, C. Zanca, and Furnari lab members for discussions and helpful suggestions. We are grateful to the IGM Genomics Center, University of California San Diego for conducting RNA sequencing, digital karyotyping, single-cell RNA sequencing and whole genome sequencing. We thank the Center for Advanced Laboratory Medicine, University 
of California San Diego for assistance with immunohistochemistry. We thank M. F. Camargo for providing graphical work. This work was supported by National Institutes of Health (NIH) R01NS080939 (F.B.F.) and R01HL137223 (G.W.Y.), the Defeat GBM Research Collaborative, a subsidiary of the National Brain Tumor Society (F.B.F., and P.S.M.), Ruth L. Kirschstein Institutional National Research Award T32 GM008666 (A.D.P.), grants from National Institute of Neurological Disorders and Stroke NS73831 (P.S.M.), the Ben and Catherine Ivy Foundation (P.S.M.), and National Institutes of Health P30CA023100 (IGM Genomics Center). I.A.C. is a San Diego IRACDA Fellow supported by NIH/NIGMS K12 GM068524 Award.

\section{AUTHOR CONTRIBUTIONS}

T.K., Se.M., G.W.Y., and F.B.F conceived and designed the study. T.K., J.A.B., A.D.P., K.M.T., F.M.H., S.S., J.M., Sh.M., and A.D.B. conducted the experiments. I.A.C., N.D.N., and M.D.A. performed bioinformatics analyses. J.R., K.A.F., V.B., C.C.C., and P.S.M. supervised parts of the study. T.K., I.A.C., G.W.Y. and F.B.F. wrote the manuscript with inputs from other authors. G.W.Y and F.B.F supervised all aspects of the study.

\section{COMPETING INTERESTS}

P.S.M. is co-founder of Pretzel Therapeutics, Inc. He has equity and serves as a consultant for the company. P.S.M. also did a one-time consultation for Abide Therapeutics, Inc. G.W.Y. is co-founder, member of the Board of Directors, equity holder, and paid consultant for Eclipse BioInnovations. The terms of these arrangements have been reviewed and approved by the University of California, San Diego in accordance with its conflict of interest policies. The authors declare no other competing financial interests. 


\section{DATA AVAILABILITY}

RNA-seq data and single-cell RNA-seq data that support the findings of this study (Fig. 4, 5, and 7, Supplementary Fig. 7-10, and 12-14) have been deposited at the Gene Expression Omnibus, accession numbers are GSE133479 and GSE133509. 


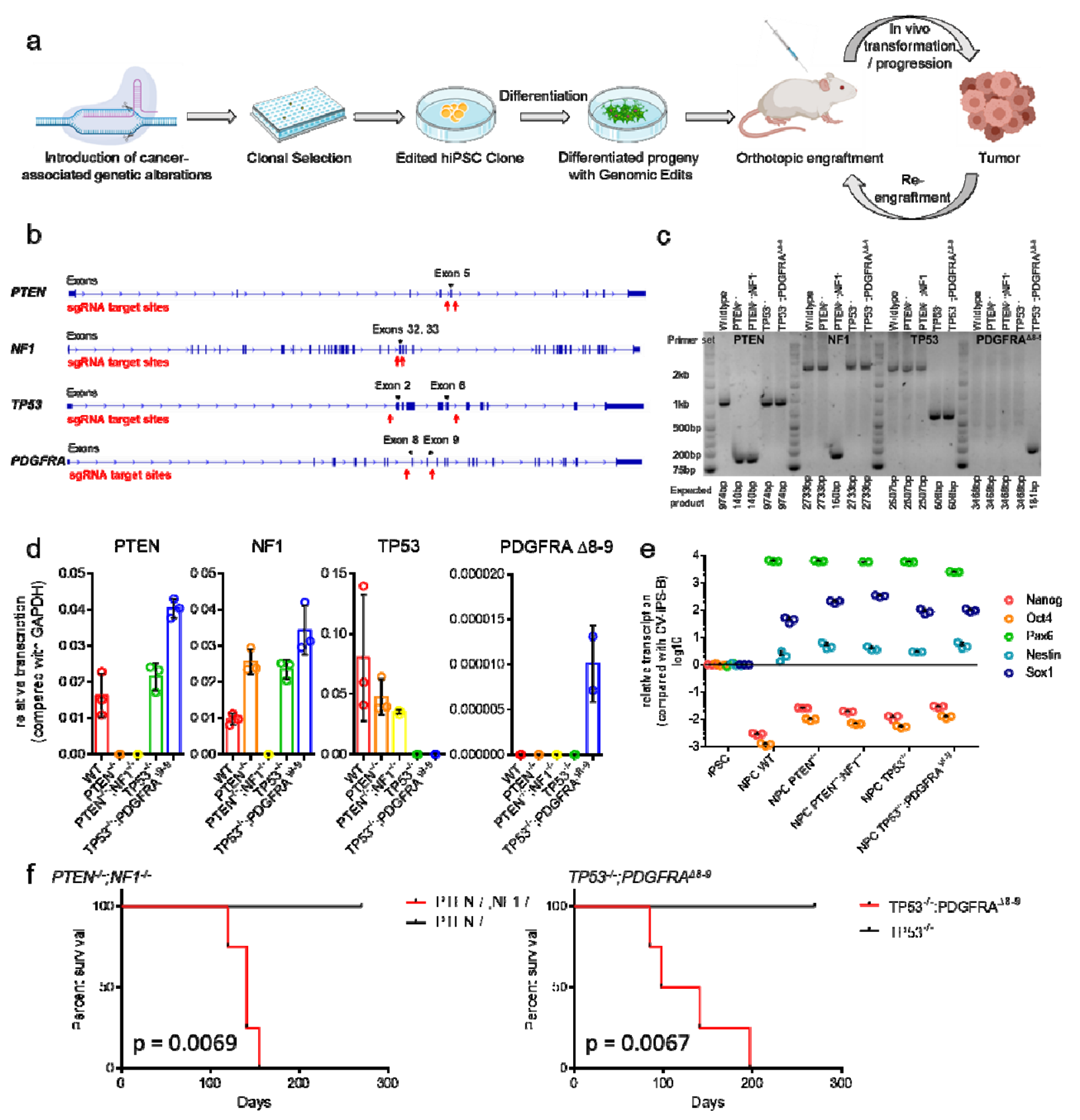

Fig. 1. Different iHGG models derived from edited human iPSCs. (a) Schema of iHGG

generation. (b) Designs for gene editing indicating placement of sgRNAs. (c) Genotyping PCR and (d) Semi-quantitative RT-qPCR evaluating designated edits. Data are representative of three replicates, $\mathrm{n}=3$. Data are represented as mean $\pm \mathrm{SD}$. (e) RT-qPCR results of markers for iPSCs and NPCs. Data are representative of three replicates, $n=3$. Data are represented as mean \pm SD. 
bioRxiv preprint doi: https://doi.org/10.1101/576009; this version posted October 25,2019 . The copyright holder for this preprint (which was not certified by peer review) is the author/funder. All rights reserved. No reuse allowed without permission.

(f) Kaplan-Meier curves showing survival of mice engrafted with (left) $P T E N^{-/}$NPCs, $P T E N^{-}$

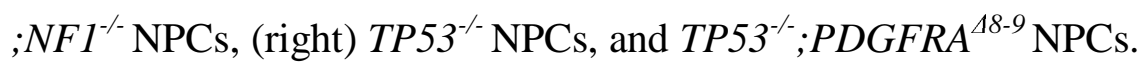




\section{PTEN ${ }^{-\infty} ; N F 1^{--}$}

a
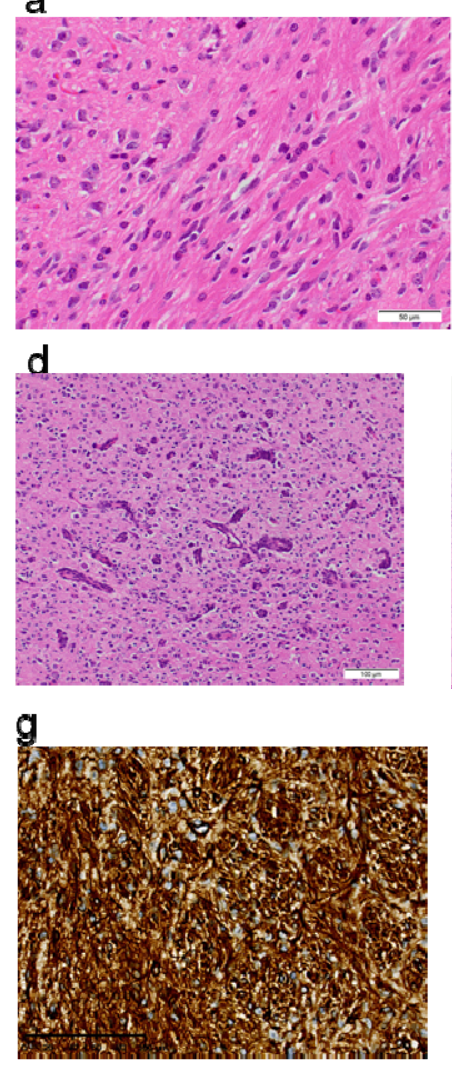

b
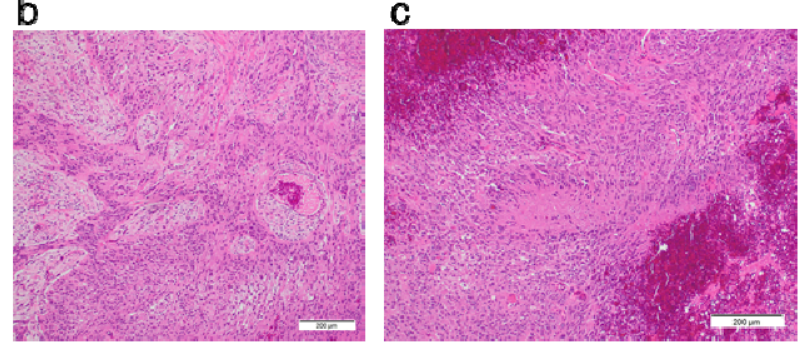

f

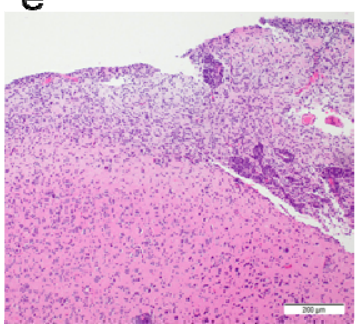

h

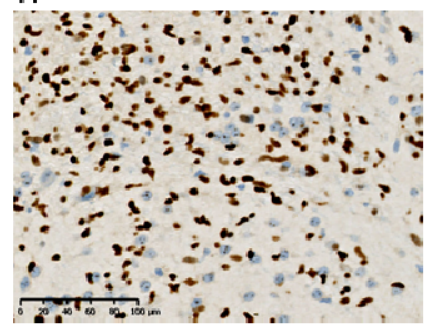

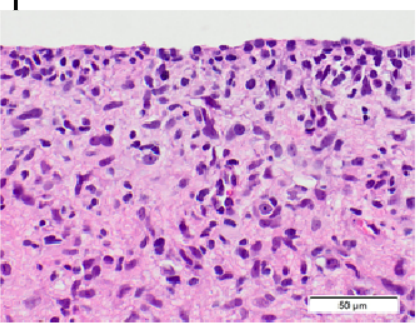

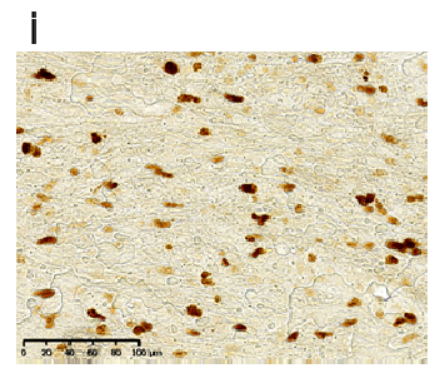

\section{TP53\%;PDGFRA $48-8$}
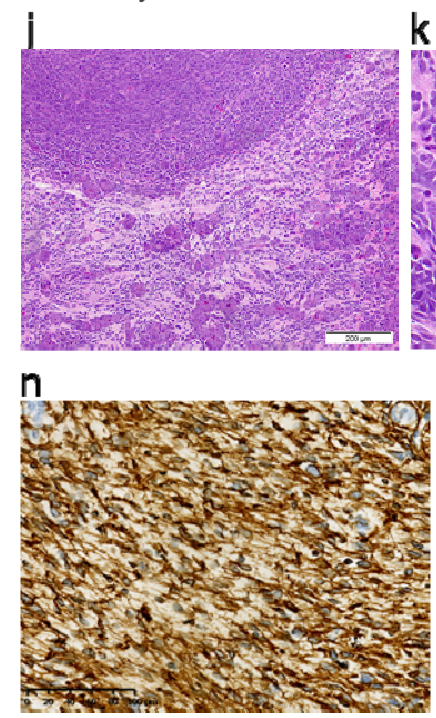
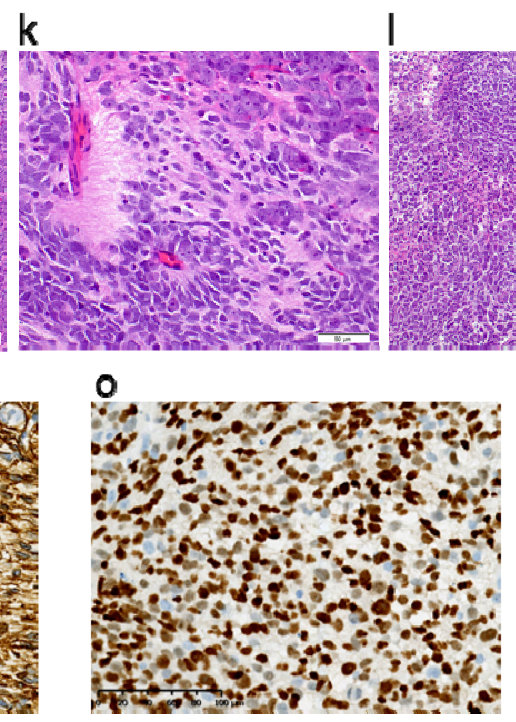

m
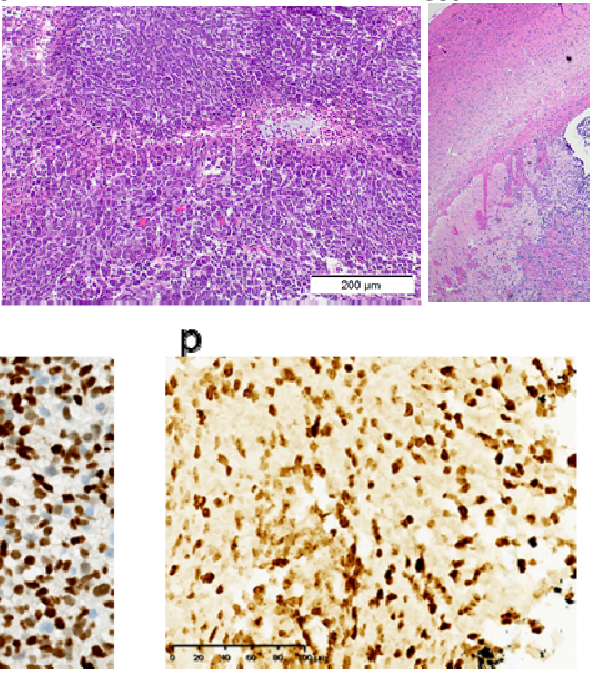

Fig. 2. Histology of iHGGs. (a-f) H\&E staining of $P T E N^{-\alpha} ; N F 1^{-/}$iHGGs showing (a) a region

of hypercellularity infiltrated by irregular, elongated to angulated tumor cells with occasional 
mitoses, (b) biphasic dense (glial) and loose (mesenchymal/sarcoma) morphologies, typical of gliosarcoma, (c) necrosis (central pink zone) with peripheral "pseudopalisading" of cells around the necrotic center, (d) vascular endothelial proliferation, (e) rupture through the pial surface, and consequently subarachnoid spread (upper right), and (f) "secondary structures" typical of glioma, including perineuronal satellitosis and subpial accumulation of tumor cells. (g) GFAP, (h) Olig2, (i) Ki-67 staining of $P T E N^{--} ; N F 1^{-/}$iHGGs. (j-m) H\&E staining of TP53 ${ }^{-/} ; P D G F R A^{48-9}$ iHGGs showing (j) nodular growth of a primitive neuronal component (dark purple) intermingled with glial component (pink), $(\mathrm{k})$ rosettes with neuropil-like texture in a primitive neuronal component, (l) a serpiginous zone of pseudopalisading necrosis, and (m) a tumor rupture through ependyma illustrating intraventricular growth. (n) GFAP, (o) Olig2, (p) Ki-67 staining of TP53\% ;PDGFRA ${ }^{48-9}$ iHGGs. 

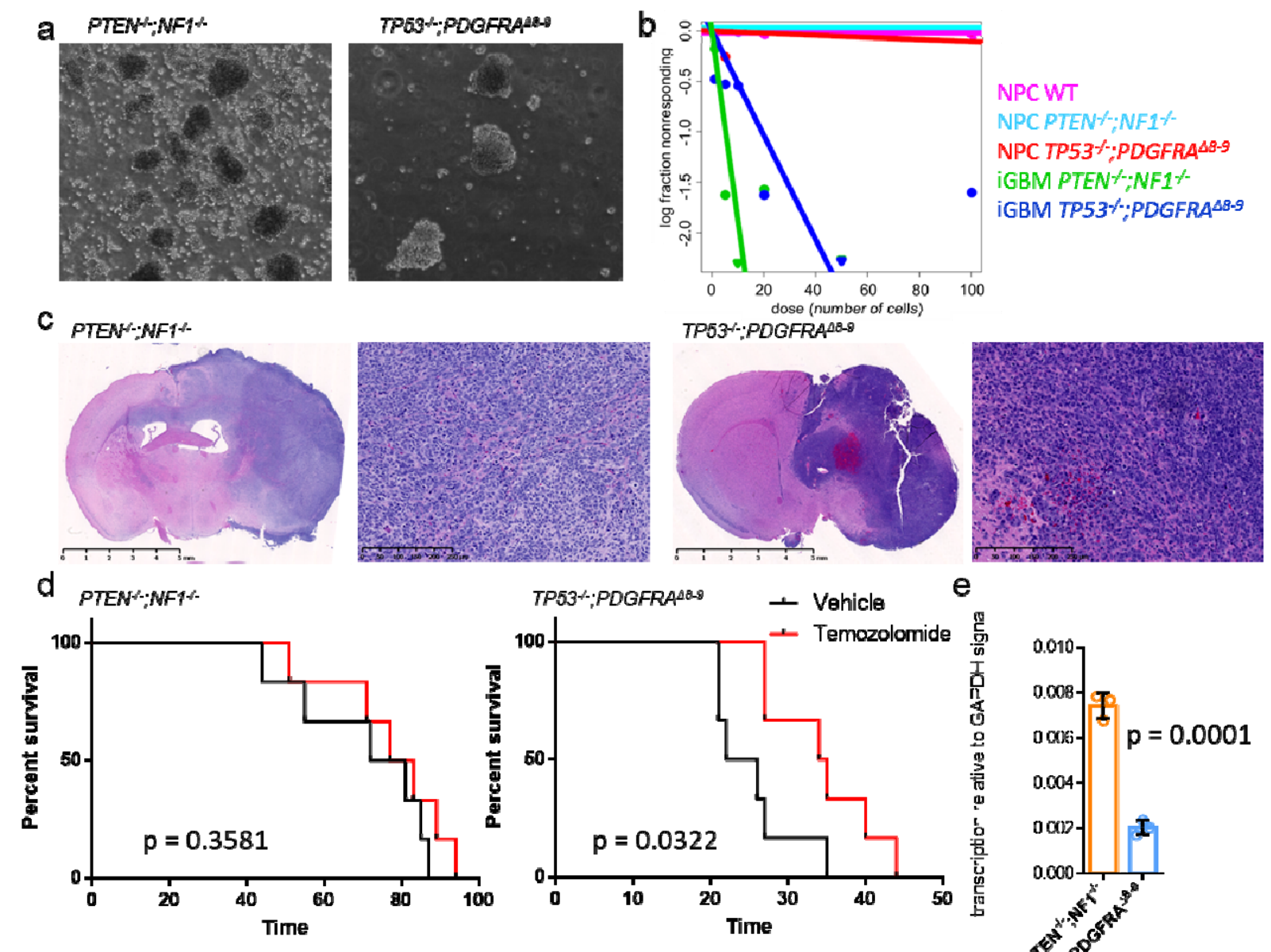

e

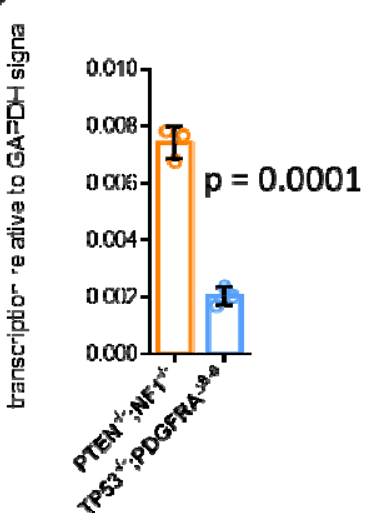

Fig. 3. Cells from iHGG models can be cultured in vitro and re-engrafted to form

secondary tumors with different drug response. (a) iHGG spheres obtained by maintaining iHGG tumor cells in neurosphere culture conditions. (b) Extreme limiting dilution analysis of input NPCs and tumor-derived iHGG sphere cells. (c) H\&E staining of secondary tumors generated from re-engraftment of primary iHGG spheres. (d) In vivo survival assays of mice orthotopically engrafted with primary iHGG sphere cells upon treatment either with vehicle or temozolomide. (e) MGMT expression levels in iHGG cells analyzed by semi-quantitative RTqPCR. Data are representative of three replicates, $n=3$. Data are represented as mean $\pm S D$, analyzed by unpaired t-test. 
bioRxiv preprint doi: https://doi.org/10.1101/576009; this version posted October 25, 2019. The copyright holder for this preprint (which was not certified by peer review) is the author/funder. All rights reserved. No reuse allowed without permission. 
bioRxiv preprint doi: https://doi.org/10.1101/576009; this version posted October 25,2019 . The copyright holder for this preprint (which was not certified by peer review) is the author/funder. All rights reserved. No reuse allowed without permission.

a

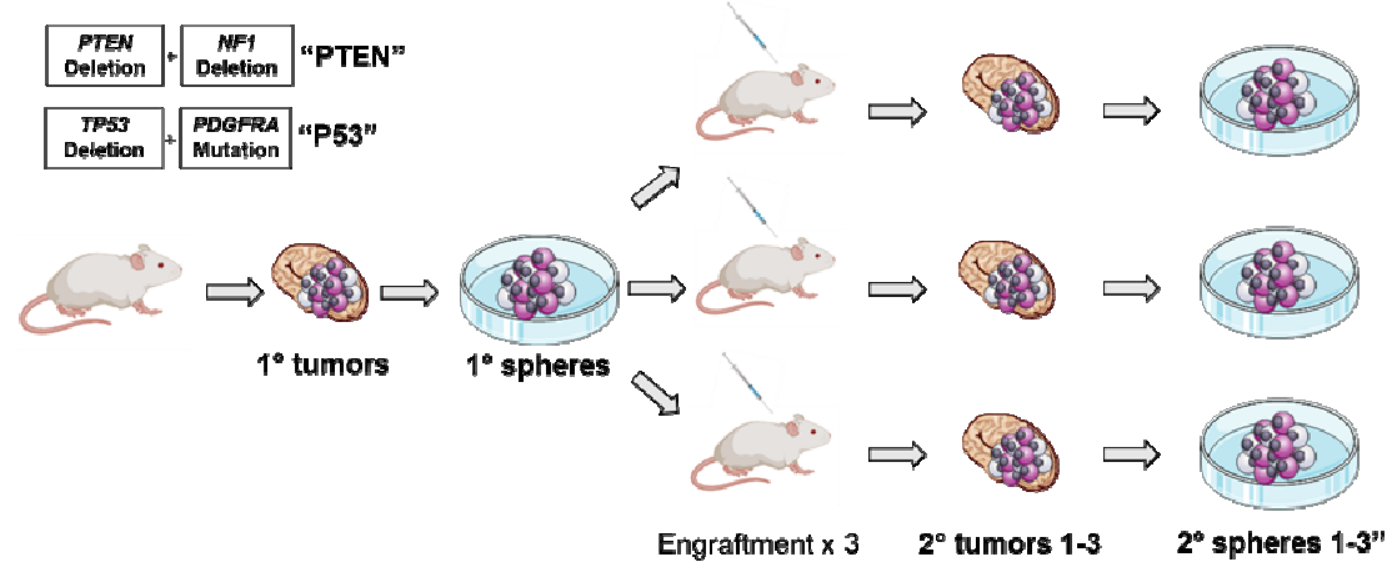

b

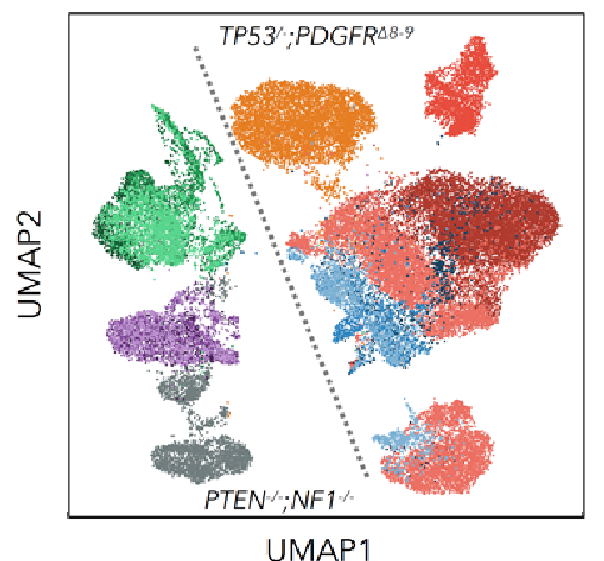

- PTEN $1^{\circ}$ Sphere

- PTEN $2^{3}$ Tumór-1

- PTEN $2^{\circ}$ Tumor-2

- PTEN $2^{\circ}$ Tumor-3

- PTEN $2^{\circ}$ Sphere-1

- PTEN $2^{\circ}$ Sphere-2

- PTEN $2^{\circ}$ Sphere-3

- P53 $1^{\circ}$ Sphere

- P53 $2^{8}$ Tumór-1

- P53 $2^{3}$ Tumor-2

- P53 $2^{\circ}$ Tumor-3

- P53 $2^{\circ}$ Sphere-1

- P53 $2^{\circ}$ Sphere-2

- P53 $2^{\circ}$ Sphere-3 c

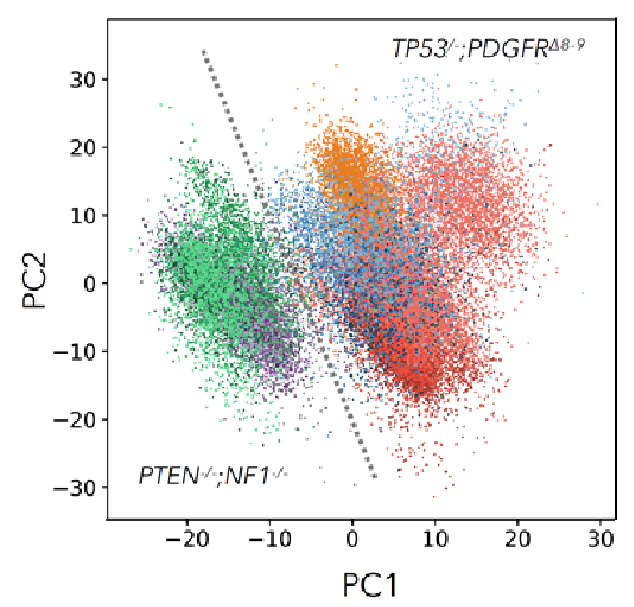

d

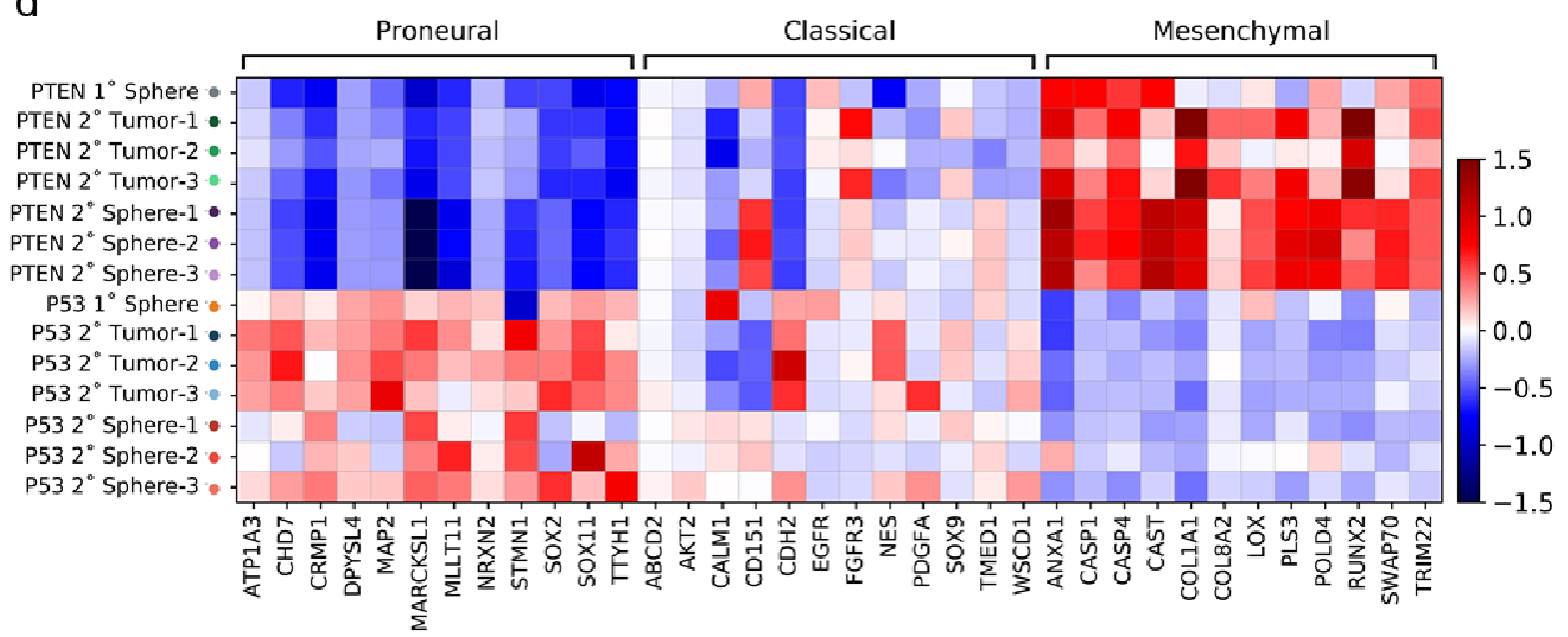


Fig. 4. iGBM models present inter-tumor heterogeneities and divergent transcriptomes driven by molecular subtypes. (a) Schema of sc-RNA seq analysis of iGBMs. (b) Uniform Manifold Approximation and Projection (UMAP) analysis of all sequenced samples. (c)

Principal component analysis of all sequenced samples. (d) Heatmap of GBM molecular subtype analysis based on average gene expression of individual cells in each sample for a manually curated gene list based on ${ }^{16}$. 


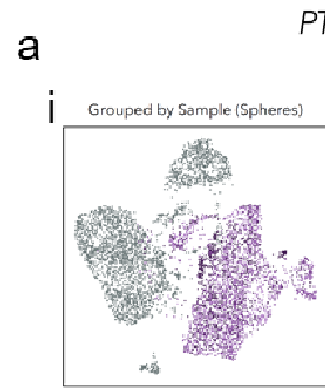

PTEN - NFF1

Spheres
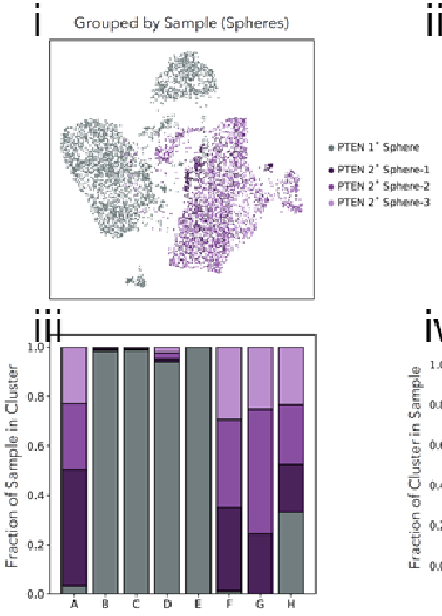

ii Grouped by Expression Cluster

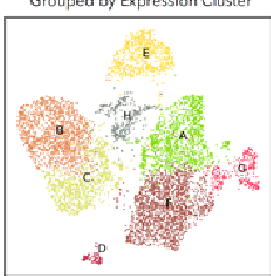

iv

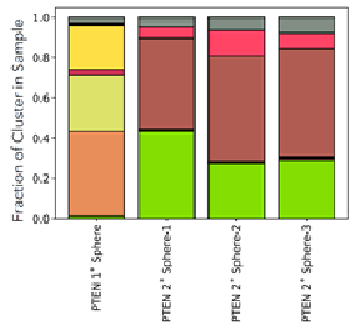

C

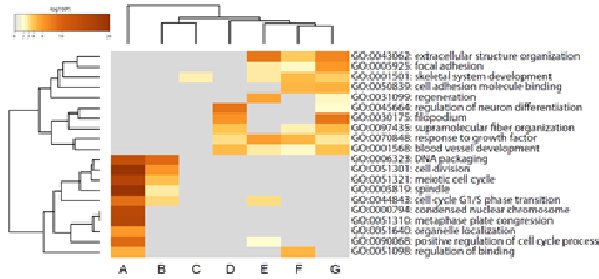

b
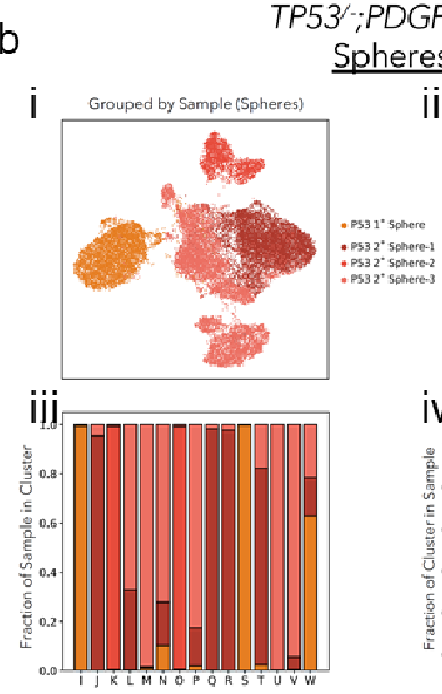

iv
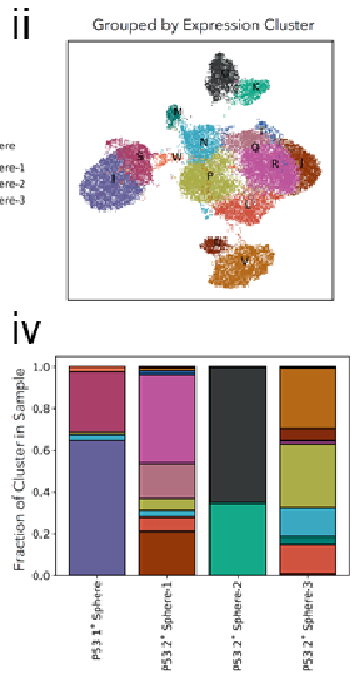

d

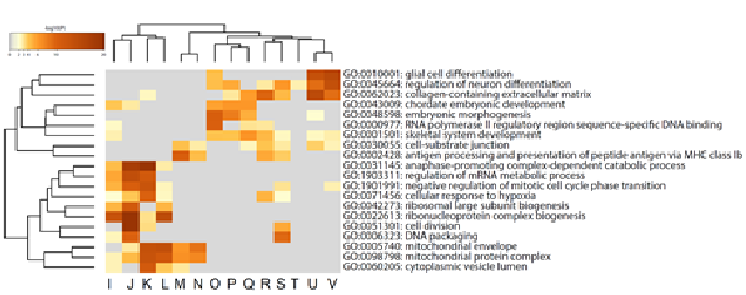

Fig. 5. Genetically distinct iHGG models present different patterns of longitudinal

evolution. (a) $P T E N^{--} ; N F 1^{-/}$and (b) of $T P 53^{-/} ; P D G F R A^{48-9}$ primary and secondary spheres. i.

UMAP plot color-coded by samples or ii. by Louvain clustering. iii. Sample distribution found in each Louvain cluster, color-coded by sample identity and iv. Louvain cluster distribution per sample, color-coded by cluster identity. Clustered heatmaps of enriched GO terms extracted from differentially expressed genes of each Louvain cluster in (c) $P T E N^{--} ; N F 1^{-/-}$and (d) $T P 53^{-/-}$ ;PDGFRA $A^{48-9}$. Color scale represents statistical significance. Gray color indicates a lack of significance. 
a

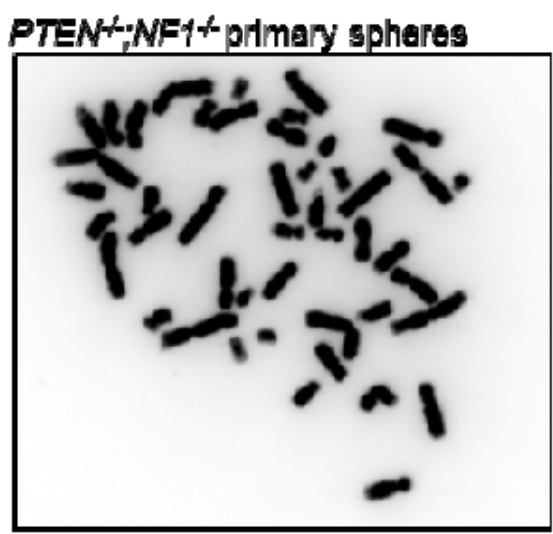

c

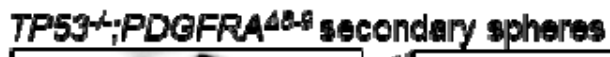

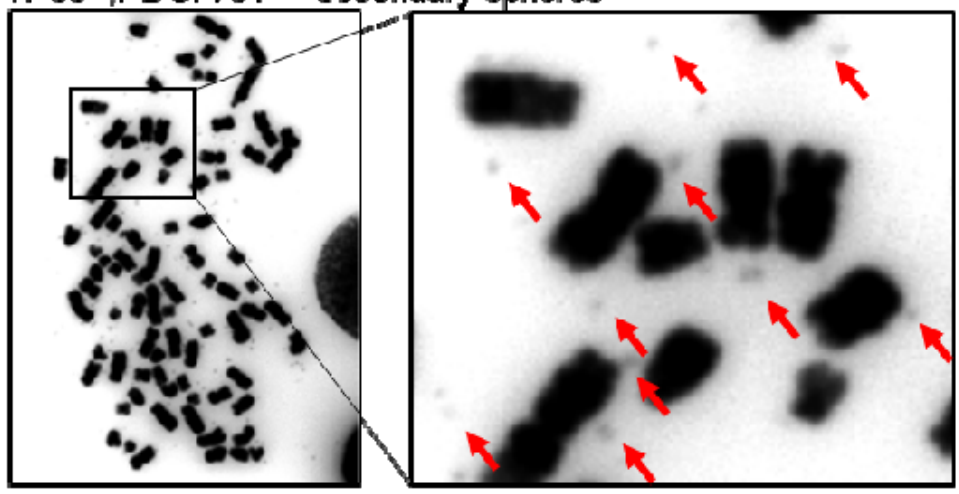

b

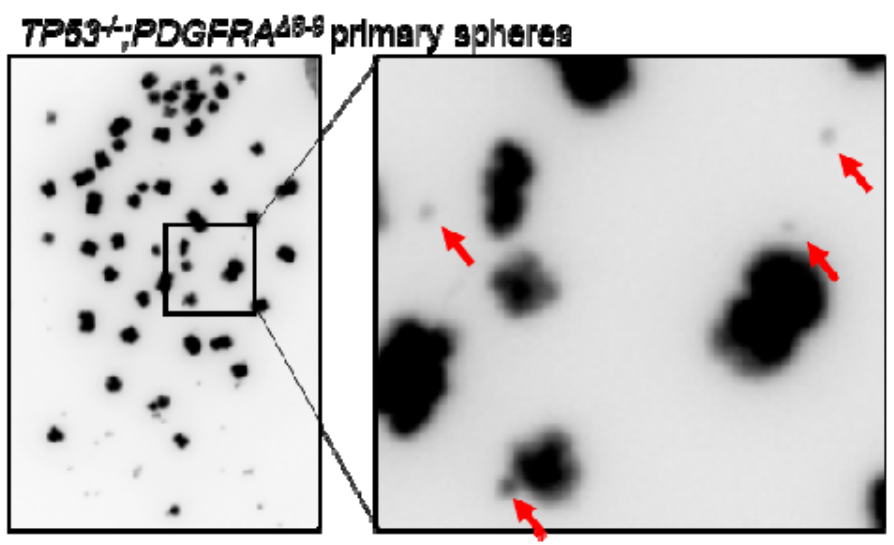

d

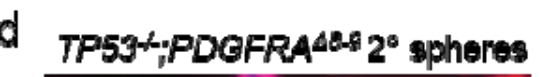

e

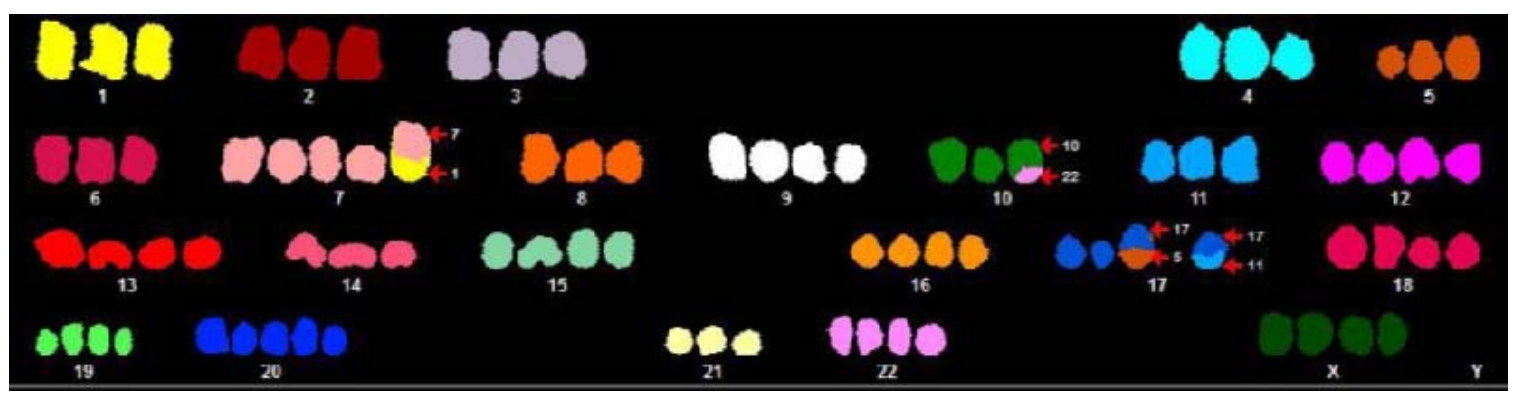

Fig. 6. TP53 ${ }^{-/}$;PDGFRA ${ }^{48-9}$ iHGG shows prominent karyotype abnormalities accompanied by extrachromosomal DNA. (a) DAPI staining of $P T E N^{-} ; N F 1^{-\nsim}$ primary iHGG cells. (b) DAPI staining of $T P 53^{--} ; P D G F R A^{48-9}$ primary iHGG cells. Red arrows indicate ecDNA. (c) DAPI staining of $T P 53^{--} ; P D G F R A^{48-9}$ secondary iHGG cells. Red arrows indicate ecDNA. (d) EdU labeling of chromosomes and ecDNA in a metaphase spread of $T P 53^{-/} ; P D G F R A^{48-9}$ secondary iGBM. (e) Spectral karyotyping analysis of TP53 $3^{--}$;PDGFRA ${ }^{\Delta 8-9}$ iHGG cells. 
bioRxiv preprint doi: https://doi.org/10.1101/576009; this version posted October 25, 2019. The copyright holder for this preprint (which was not certified by peer review) is the author/funder. All rights reserved. No reuse allowed without permission. 

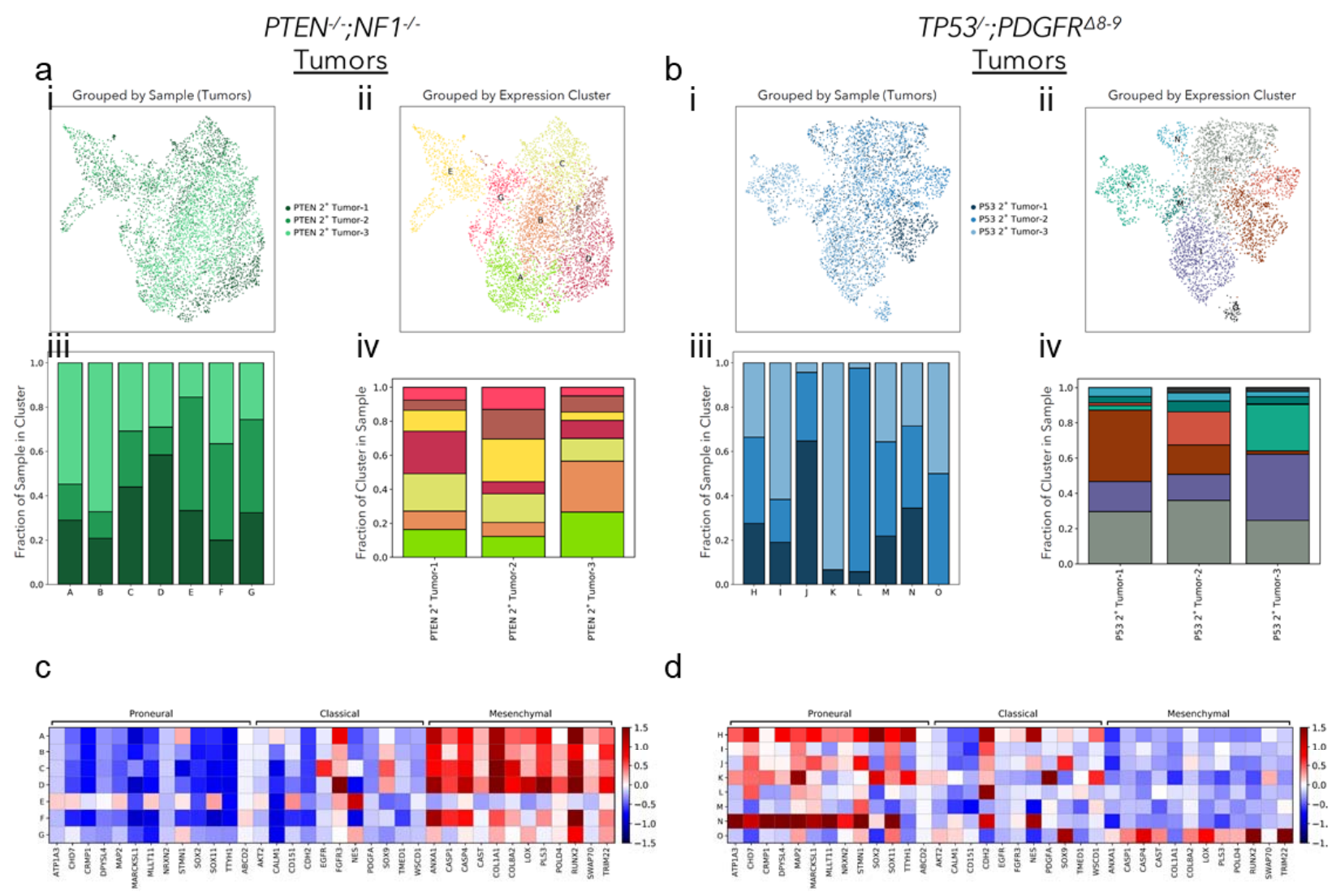

d
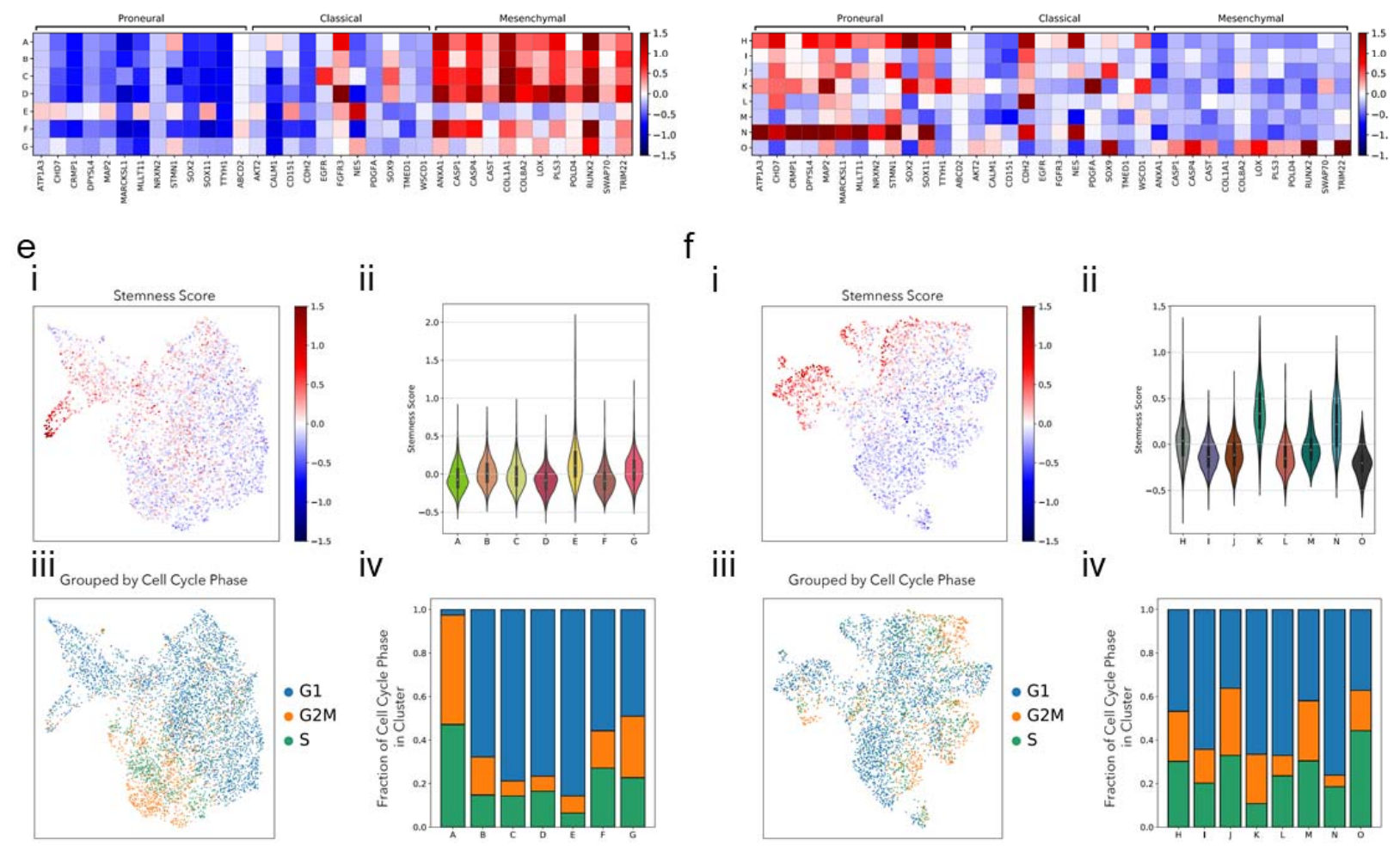

iv
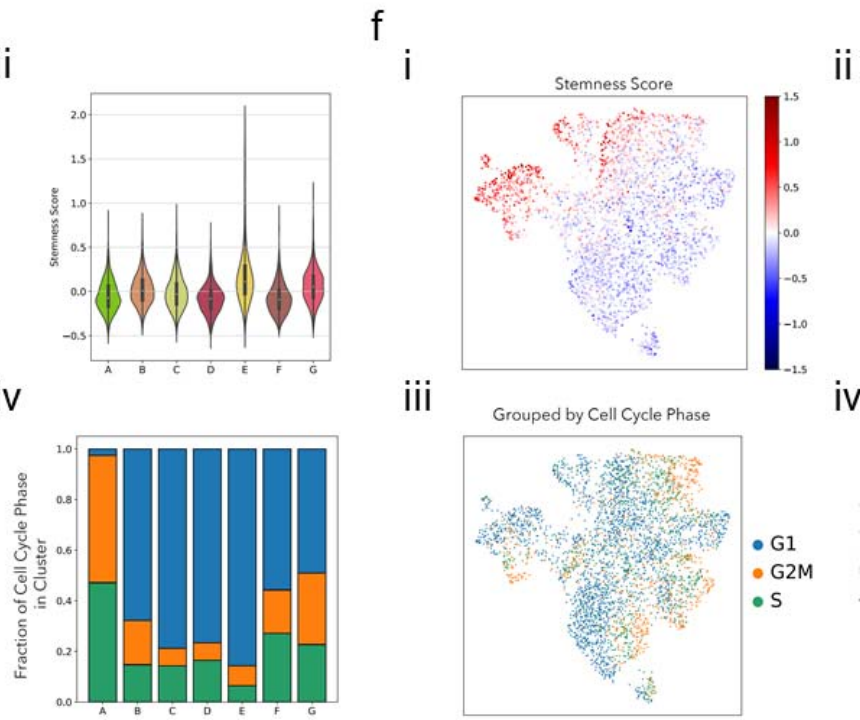

iii
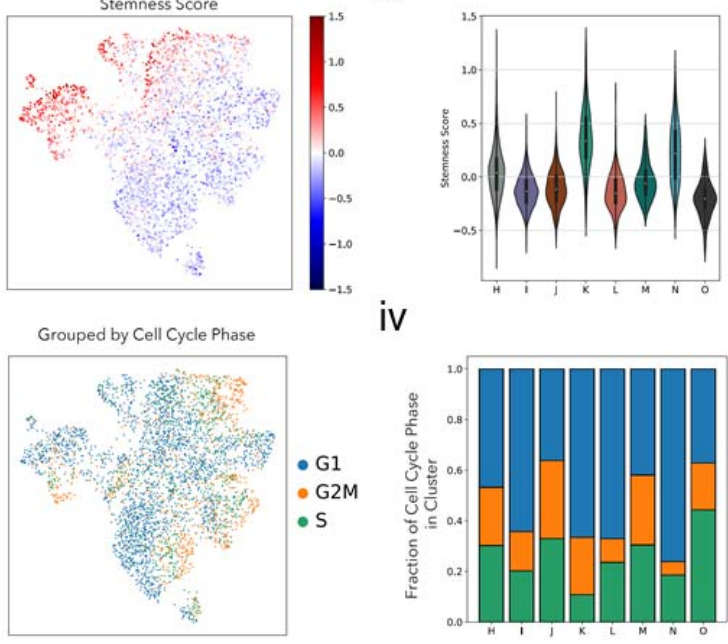

iv

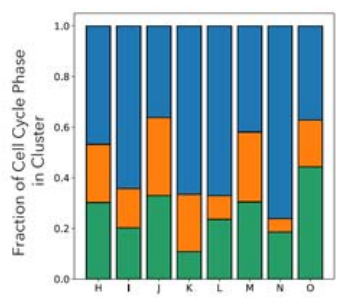

Fig. 7. iHGG tumors confirm features characteristic of patient tumor samples. (a) $P T E N^{/-}$

;NF1 $1^{-/-}$and (b) of $T P 53^{-/-} ; P D G F R A^{48-9}$ secondary tumors. i. UMAP plot color-coded by samples or ii. by Louvain clustering. iii. Sample distribution found in each Louvain cluster, color-coded 
by sample identity and iv. Louvain cluster distribution per sample, color-coded by cluster identity. GBM molecular subtype analysis based on average gene expression of individual cells in each Louvain cluster for (c) $P T E N^{-/} ; N F 1^{-/}$and (d) $T P 53^{-/} ; P D G F R A^{48-9}$ tumors. For (e) $\mathrm{PTEN}^{/-} ; \mathrm{NFI}^{-/-}$and (f) $\mathrm{TP}^{-3^{-/}} ; \mathrm{PDGFRA}^{48-9}$ tumors, stemness scores were calculated for each cell and results overlaid on a UMAP plot (i) or summarized as violin plots for each cluster (ii). Cells were categorized based on their cell cycle status (G1, G2M or S), overlaid on a UMAP plot (iii) and their distribution was calculated for each Louvain cluster (iv). 


\section{REFERENCES}

1 Cloughesy, T. F., Cavenee, W. K. \& Mischel, P. S. Glioblastoma: from molecular pathology to targeted treatment. Annu Rev Pathol 9, 1-25, doi:10.1146/annurev-pathol011110-130324 (2014).

2 Stylli, S. S., Luwor, R. B., Ware, T. M., Tan, F. \& Kaye, A. H. Mouse models of glioma. J Clin Neurosci 22, 619-626, doi:10.1016/j.jocn.2014.10.013 (2015).

3 Chow, L. M. et al. Cooperativity within and among Pten, p53, and Rb pathways induces high-grade astrocytoma in adult brain. Cancer Cell 19, 305-316, doi:10.1016/j.ccr.2011.01.039 (2011).

4 Lenting, K., Verhaak, R., Ter Laan, M., Wesseling, P. \& Leenders, W. Glioma: experimental models and reality. Acta neuropathologica 133, 263-282 (2017).

5 Rich, J. N. et al. A genetically tractable model of human glioma formation. Cancer Res 61, 3556-3560 (2001).

6 Sonoda, Y. et al. Formation of intracranial tumors by genetically modified human astrocytes defines four pathways critical in the development of human anaplastic astrocytoma. Cancer Res 61, 4956-4960 (2001).

$7 \quad$ Neftel, C. et al. An integrative model of cellular states, plasticity, and genetics for Glioblastoma. Cell 178, 835-849. e821 (2019).

8 Wang, Q. et al. Tumor evolution of glioma-intrinsic gene expression subtypes associates with immunological changes in the microenvironment. Cancer cell 32, 42-56. e46 (2017).

9 Patrizii, M., Bartucci, M., Pine, S. R. \& Sabaawy, H. E. Utility of glioblastoma patientderived orthotopic xenografts in drug discovery and personalized therapy. Frontiers in oncology 8, 23 (2018). 
10 Hockemeyer, D. \& Jaenisch, R. Induced Pluripotent Stem Cells Meet Genome Editing. Cell Stem Cell 18, 573-586, doi:10.1016/j.stem.2016.04.013 (2016).

11 Drost, J. et al. Sequential cancer mutations in cultured human intestinal stem cells. Nature 521, 43-47, doi:10.1038/nature14415 (2015).

12 Matano, M. et al. Modeling colorectal cancer using CRISPR-Cas9-mediated engineering of human intestinal organoids. Nat Med 21, 256-262, doi:10.1038/nm.3802 (2015).

13 Duan, S. et al. PTEN deficiency reprogrammes human neural stem cells towards a glioblastoma stem cell-like phenotype. Nat Commun 6, 10068, doi:10.1038/ncomms10068 (2015).

14 Cong, L. et al. Multiplex genome engineering using CRISPR/Cas systems. Science 339, 819-823, doi:10.1126/science.1231143 (2013).

15 Jinek, M. et al. RNA-programmed genome editing in human cells. Elife 2, e00471, doi:10.7554/eLife.00471 (2013).

16 Verhaak, R. G. et al. Integrated genomic analysis identifies clinically relevant subtypes of glioblastoma characterized by abnormalities in PDGFRA, IDH1, EGFR, and NF1. Cancer Cell 17, 98-110, doi:10.1016/j.ccr.2009.12.020 (2010).

17 Brennan, C. W. et al. The somatic genomic landscape of glioblastoma. Cell 155, 462-477, doi:10.1016/j.cell.2013.09.034 (2013).

18 Ozawa, T. et al. PDGFRA gene rearrangements are frequent genetic events in PDGFRAamplified glioblastomas. Genes Dev 24, 2205-2218, doi:10.1101/gad.1972310 (2010).

19 Reinhardt, P. et al. Derivation and expansion using only small molecules of human neural progenitors for neurodegenerative disease modeling. PLoS One 8, e59252, doi:10.1371/journal.pone.0059252 (2013). 
20 Pauli, C. et al. Personalized In Vitro and In Vivo Cancer Models to Guide Precision Medicine. Cancer Discov 7, 462-477, doi:10.1158/2159-8290.CD-16-1154 (2017).

21 Akhavan, D. et al. De-repression of PDGFRbeta transcription promotes acquired resistance to EGFR tyrosine kinase inhibitors in glioblastoma patients. Cancer Discov $\mathbf{3}$, 534-547, doi:10.1158/2159-8290.CD-12-0502 (2013).

$22 \mathrm{Hu}$, Y. \& Smyth, G. K. ELDA: extreme limiting dilution analysis for comparing depleted and enriched populations in stem cell and other assays. J Immunol Methods 347, 70-78, doi:10.1016/j.jim.2009.06.008 (2009).

23 Stupp, R. et al. Radiotherapy plus concomitant and adjuvant temozolomide for glioblastoma. N Engl J Med 352, 987-996, doi:10.1056/NEJMoa043330 (2005).

24 Hegi, M. E. et al. MGMT gene silencing and benefit from temozolomide in glioblastoma. N Engl J Med 352, 997-1003, doi:10.1056/NEJMoa043331 (2005).

25 Blough, M. D., Beauchamp, D. C., Westgate, M. R., Kelly, J. J. \& Cairncross, J. G. Effect of aberrant p53 function on temozolomide sensitivity of glioma cell lines and brain tumor initiating cells from glioblastoma. J Neurooncol 102, 1-7, doi:10.1007/s11060010-0283-9 (2011).

26 Nagel, Z. D. et al. DNA Repair Capacity in Multiple Pathways Predicts Chemoresistance in Glioblastoma Multiforme. Cancer Res 77, 198-206, doi:10.1158/0008-5472.CAN-161151 (2017).

27 Patel, A. P. et al. Single-cell RNA-seq highlights intratumoral heterogeneity in primary glioblastoma. Science 344, 1396-1401, doi:10.1126/science.1254257 (2014). 
28 Nathanson, D. A. et al. Targeted therapy resistance mediated by dynamic regulation of extrachromosomal mutant EGFR DNA. Science 343, 72-76, doi:science.1241328 [pii]10.1126/science.1241328 (2014).

29 Turner, K. M. et al. Extrachromosomal oncogene amplification drives tumour evolution and genetic heterogeneity. Nature 543, 122-125, doi:nature21356 [pii]10.1038/nature21356 (2017).

30 deCarvalho, A. C. et al. Discordant inheritance of chromosomal and extrachromosomal DNA elements contributes to dynamic disease evolution in glioblastoma. Nat Genet 50, 708-717, doi:10.1038/s41588-018-0105-0 (2018).

31 Herting, C. et al. Genetic driver mutations define the expression signature and microenvironmental composition of high $\square$ grade gliomas. Glia $\mathbf{6 5}, 1914-1926$ (2017).

32 Jun, H. J. et al. A PDGFR $\alpha$-driven mouse model of glioblastoma reveals a stathmin1mediated mechanism of sensitivity to vinblastine. Nature communications $\mathbf{9}, 3116$ (2018).

33 Ozawa, T. et al. Most human non-GCIMP glioblastoma subtypes evolve from a common proneural-like precursor glioma. Cancer cell 26, 288-300 (2014).

34 Huang, M. et al. Engineering Genetic Predisposition in Human Neuroepithelial Stem Cells Recapitulates Medulloblastoma Tumorigenesis. Cell stem cell (2019).

35 Bian, S. et al. Genetically engineered cerebral organoids model brain tumor formation. Nat Methods 15, 631-639, doi:10.1038/s41592-018-0070-7 (2018).

36 Bachoo, R. M. et al. Epidermal growth factor receptor and Ink4a/Arf: convergent mechanisms governing terminal differentiation and transformation along the neural stem cell to astrocyte axis. Cancer Cell 1, 269-277 (2002). 
37 Giannini, C. et al. Patient tumor EGFR and PDGFRA gene amplifications retained in an invasive intracranial xenograft model of glioblastoma multiforme. Neuro Oncol 7, 164176, doi:10.1215/S1152851704000821 (2005).

38 Zou, H. et al. Double minute amplification of mutant PDGF receptor alpha in a mouse glioma model. Sci Rep 5, 8468, doi:10.1038/srep08468 (2015).

39 Camacho, C. V. et al. DNA double-strand breaks cooperate with loss of Ink4 and Arf tumor suppressors to generate glioblastomas with frequent Met amplification. Oncogene 34, 1064-1072, doi:10.1038/onc.2014.29 (2015).

40 Gore, A. et al. Somatic coding mutations in human induced pluripotent stem cells. Nature 471, 63-67, doi:10.1038/nature09805 (2011).

41 Ran, F. A. et al. Genome engineering using the CRISPR-Cas9 system. Nat Protoc 8, 2281-2308, doi:10.1038/nprot.2013.143 (2013).

42 Dobin, A. et al. STAR: ultrafast universal RNA-seq aligner. Bioinformatics 29, 15-21, doi:10.1093/bioinformatics/bts635 (2013).

43 Harrow, J. et al. GENCODE: the reference human genome annotation for The ENCODE Project. Genome Res 22, 1760-1774, doi:10.1101/gr.135350.111 (2012).

44 Anders, S., Pyl, P. T. \& Huber, W. HTSeq--a Python framework to work with highthroughput sequencing data. Bioinformatics 31, 166-169, doi:10.1093/bioinformatics/btu638 (2015).

45 Anders, S., Reyes, A. \& Huber, W. Detecting differential usage of exons from RNA-seq data. Genome Res 22, 2008-2017, doi:10.1101/gr.133744.111 (2012). 
46 Love, M. I., Huber, W. \& Anders, S. Moderated estimation of fold change and dispersion for RNA-seq data with DESeq2. Genome Biol 15, 550, doi:10.1186/s13059-014-0550-8 (2014).

47 Anders, S. \& Huber, W. Differential expression analysis for sequence count data. Genome Biol 11, R106, doi:10.1186/gb-2010-11-10-r106 (2010).

48 Wolf, F. A., Angerer, P. \& Theis, F. J. SCANPY: large-scale single-cell gene expression data analysis. Genome Biol 19, 15, doi:10.1186/s13059-017-1382-0 (2018).

49 Zhou, Y. et al. Metascape provides a biologist-oriented resource for the analysis of systems-level datasets. Nat Commun 10, 1523, doi:10.1038/s41467-019-09234-6 (2019). 


\section{ONLINE METHODS}

Cell culture. Experiments using human pluripotent stem cells were conducted under the regulations of the UCSD Human Research Protections Program, project number 151330ZX. Human iPS cells, CV-iPS-B cells ${ }^{40}$, were cultured on plates coated with Matrigel hESCQualified Matrix (Corning) in mTeSR1 media (Stemcell Technologies). NPCs were cultured on Matrigel-coated plates in NPC maintenance media containing DMEM/F12 with GlutaMAX (Thermo Fisher Scientific), 1 x N-2 supplement (Thermo Fisher Scientific), 1 x B-27 supplement (Thermo Fisher Scientific), 50mM ascorbic acid (Tocris), $3 \mu \mathrm{M}$ CHIR99021 (Tocris) and $0.5 \mu \mathrm{M}$ purmorphamine (Tocris). Sphere cells were cultured in suspension in DMEM/F12 with GlutaMAX (Thermo Fisher Scientific) with 1 x B-27 supplement, 20 ng/mL EGF (Stemcell Technologies) and $20 \mathrm{ng} / \mathrm{mL}$ bFGF (Stemcell Technologies).

Generation and validation of genetically engineered hiPSC clones. A plasmid, pSpCas9(BB)-2A-GFP or px458, which expresses Cas9-T2A-GFP and sgRNA ${ }^{41}$, was purchased from Addgene (Plasmid \#48138). The designated sgRNA sequences for each of the targeted genes were cloned into px458 using combinations of top and bottom oligonucleotides listed below.

PTEN-intron 4-top: 5'-CACCGGAATTTACGCTATACGGAC-3', PTEN-intron 4-bottom: 5'-AAACGTCCGTATAGCGTAAATTCC-3', PTEN-intron 5-top: 5' -CACCGAACAAGATCTGAAGCTCTAC-3', PTEN-intron 5-bottom: 5'-AAACGTAGAGCTTCAGATCTTGTTC-3', TP53-intron 1-top: 5' -CACCGGGTTGGAAGTGTCTCATGC-3', TP53-intron 1-bottom: 5'-AAACGCATGAGACACTTCCAACCC-3', 
TP53-intron 6-top: 5'-CACCGCATCTCATGGGGTTATAGGG-3', TP53-intron 6-bottom: 5'-AAACCCCTATAACCCCATGAGATGC-3', NF1-intron 31-top: 5'-CACCGATAGCACTCTTCCCGAGCTA-3', NF1-intron 31-bottom: 5'-AAACTAGCTCGGGAAGAGTGCTATC-3', NF1-intron 33-top: 5'-CACCGCTTTGGGGAGGTCTTTCGTC-3', NF1-intron 33-bottom: 5'-AAACGACGAAAGACCTCCCCAAAGC-3', PDGFRA-intron 7-top: 5'-CACCGATTTGTATGTAGCGGTCTGC-3', PDGFRA-intron 7-bottom: 5'-AAACGCAGACCGCTACATACAAATC-3', PDGFRA-intron 9-top: 5'-CACCGCCACGGGAACACTCTAAGA-3', PDGFRA-intron 9-bottom: 5'-AAACTCTTAGAGTGTTCCCGTGGC-3'.

Each of top and bottom oligonucleotides were phosphorylated and annealed by incubating 10 $\mu \mathrm{M}$ each of oligonucleotides, 1 x T4 DNA ligase buffer (New England Biolabs), 5U T4 polynucleotide kinase (New England Biolabs) at $37^{\circ} \mathrm{C}$ for 30 minutes, $95^{\circ} \mathrm{C}$ for five minutes and by cooling down to $25^{\circ} \mathrm{C}$ at $0.1^{\circ} \mathrm{C} /$ second using a thermocycler. Annealed oligonucleotides were cloned into px458 by incubating $25 \mathrm{ng}$ px458, 1uM annealed oligonucleotides, $1 \mathrm{X}$ CutSmart buffer (New England Biolabs), 1 mM ATP (New England Biolabs), 10U BBSI-HF (New England Biolabs) and 200U T4 ligase (New England Biolabs) at $37^{\circ} \mathrm{C}$ for 5 minutes, $23^{\circ} \mathrm{C}$ for five minutes for 30 cycles. Correct cloning of each sgRNA sequence was confirmed by Sanger sequencing using U6 sequencing primer: 5'-GATACAAGGCTGTTAGAGAGATAATT$3^{\prime}$.

Human iPSCs were cultured in $10 \mu \mathrm{M}$ Y-27632 RHO/ROCK pathway inhibitor for 2 hours before dissociation. The cells were dissociated to single cells using Accutase (Innovative Cell Technologies). The dissociated hiPSCs ( $1 \times 10^{6}$ cells) were resuspended in $100 \mu 1$ of 
supplemented solution of Human Stem Cell Nucleofector Kit 1 (Lonza) containing $8 \mu \mathrm{g}$ total of a combination of px458 plasmids targeting each gene and electroporated using B-016 program of Nucleofector 2b (Lonza). Electroporated hiPSCs were cultured on Matrigel-coated plates in mTeSR1 for 48 hours. GFP-positive cells were then sorted by flow cytometer (SH800, SONY) and 1-2 x $10^{4}$ sorted cells were plated on a 10-cm Matrigel-coated plate in mTeSR1. Isolated colonies were manually picked and plated in duplicated Matrigel-coated 96-well plates.

The hiPSCs clones on one of the duplicated 96-well plates were lysed using QuickExtract DNA Extraction Solution (Epicentre) and genotyping PCR was performed using Platinum Taq DNA Polymerase High Fidelity (Thermo Fisher Scientific) in $10-\mu 1$ reaction volume containing $0.2 \mu \mathrm{M}$ of each primer with the following reaction conditions: $94^{\circ} \mathrm{C}$ for $2 \mathrm{~min}, 40$ cycles of $94^{\circ} \mathrm{C}$ for $15 \mathrm{~s}$, $55^{\circ} \mathrm{C}$ for $30 \mathrm{~s}$, and $68^{\circ} \mathrm{C}$ for $4 \mathrm{~min}$. Primers used for the genotyping PCR are listed below.

PTEN-i4-f: 5'-GAGTCCTGACGAAATGTCCATG-3', PTEN-i5-r: 5'-CCTGTT TTCCAGGGACTGAG-3', NF1-i31-f: 5'-ACTCTGGAAAGGGATGGGAG-3', NF1-i33-r: 5'-CCGGCTTCAGCTTCAAAGTAG-3', TP53-i1-f: 5'-CCGATCACCTGAAGTAAGGAG-3', TP53-i6-r: 5'-CCTTAGCCTCTGTAAGCTTCAG-3', PDGFRA-i7-f: 5' -TGTACTCCTGTCCCCAGCTG-3', PDGFRA-i9-r: 5'-TCCTGAGAGTCATGGCAATG-3'.

Total RNA was extracted from the edited hiPSCs using RNeasy Plus Mini Kit (Qiagen) and was reverse transcribed using RNA to cDNA EcoDry Premix (Clontech) according to the manufacturer's instruction. Triplicate qPCR reactions containing cDNA obtained from 10ng equivalent RNA were run on a CFX96 Real Time System (Bio-Rad) to confirm designated 
targeting of the genes with the following reaction conditions: $95^{\circ} \mathrm{C}$ for $5 \mathrm{~min}, 40$ cycles of $95^{\circ} \mathrm{C}$ for $15 \mathrm{~s}, 56^{\circ} \mathrm{C}$ for $30 \mathrm{~s}$. Primer pairs were designed to span the deleted regions of each target gene. The data was normalized to GAPDH and the relative transcript levels were determined using $2^{-4} \mathrm{Ct}$ formula. Primers used for the RT-qPCR are listed below.

GAPDH-RT-f: 5'-AATTTGGCTACAGCAACAGGGTGG-3', GAPDH-RT-r: 5'-TTGATGGTACATGACAAGGTGCGG-3', PTEN-RT-f: 5'-CGAACTGGTGTAATGATATGT-3', PTEN-RT-r: 5'-CATGAACTTGTCTTCCCGT-3', NF1-RT-f: 5'-GCCACCACCTAGAATCGAAAG-3', NF1-RT-r: 5'-AGCAAGCACATTGCCGTCAC-3', TP53-RT-f: 5'-CCAAGTCTGTGACTTGCACG-3', TP53-RT-r: 5'-GTGGAATCAACCCACAGCTG-3', PDGFRA $^{\Delta 8-9}$-RT-f: 5'-GATGTGGAAAAGATTCAGGAAATAAGATG-3', PDGFRA ${ }^{\mathrm{wt}}$-RT-f: 5' -CGCCGCTTCCTGATATTGAG-3', PDGFRA-RT-r: 5'-CTCCACGGTACTCCTGTCTC-3'.

The qPCR products were visualized by agarose gel electrophoresis.

Differentiation of hiPSCs to neural progenitor cells. Generation of small molecule neural progenitor cells (smNPCs) from iPSCs was adapted from a previous study ${ }^{19}$. Briefly, human iPSCs at 70\%-80\% confluency were dissociated using accutase (Innovative Cell Technologies) and resuspended at $1 \times 10^{6}$ cells/ml in N2B27 medium (DMEM/F12 with GlutaMAX (Thermo Fisher Scientific), 1 x N-2 supplement (Thermo Fisher Scientific), 1 x B-27 supplement (Thermo Fisher Scientific), $150 \mathrm{mM}$ ascorbic acid (Tocris), and 1\% Penicillin/Streptomycin) 
supplemented with $1 \mu \mathrm{M}$ Dorsomorphin (Tocris), $10 \mu \mathrm{M}$ SB431542 (Tocris), $3 \mu \mathrm{M}$ CHIR99021, $0.5 \mu \mathrm{M}$ Purmorphamine and $5 \mathrm{mM}$ Y-26732 (Stemcell Technologies). Three million cells were transferred into one well of an uncoated 6-well tissue culture plate and incubated at $37^{\circ} \mathrm{C}, 5 \%$ $\mathrm{CO} 2$ on a shaker at $90 \mathrm{rpm}$. Uniform small EBs formed within $24 \mathrm{~h}$ and increased in size over the following days. After 48h, a full media change was performed with N2B27 medium supplemented with Dorsomorphin, SB431542, CHIR99021, and Purmorphamine. At this time, about 2/3 of EBs were either discarded or EBs were split across 3 wells of a 6-well plate to reduce the high cell density required initially to ensure uniform formation of embryoid bodies. On days 3-5, half media change was performed with fresh N2B27 media supplemented with Dorsomorphin, SB431542, CHIR99021, and Purmorphamine. On day 6, Dorsomorphin and SB431542 were withdrawn and a full media change with smNPC media (N2B27 media supplemented with $3 \mu \mathrm{M}$ CHIR99021 and $0.5 \mu \mathrm{M}$ Purmorphamine) was performed. At this stage, neuroepithelial folds were clearly visible in all EBs. On day 8, EBs were triturated by pipetting 10-15 times with a P1000 pipette and plated onto matrigel-coated $10 \mathrm{~cm}$ plates. After 3-4 days, attached EB fragments and outgrown cells were dissociated to single cells with accutase (Innovative Cell Technologies) and split at a 1:6 to 1:8 ratio onto matrigel-coated plates. After the first passage, cells were passaged at a 1:10 to 1:15 ratio every 3-6 days. For the first few passages, large flat non-smNPCs could be observed between smNPC colonies, but progressively disappeared no later than passages 3-6 in almost all cell lines. Total RNA was extracted from the differentiated smNPCs using RNeasy Plus Mini Kit and was reverse transcribed using RNA to cDNA EcoDry Premix according to the manufacturer's instruction. Triplicate qPCR reactions containing cDNA obtained from 10 ng equivalent RNA were run on a CFX96 Real Time System to confirm NPC differentiation with the following reaction conditions: 
$95^{\circ} \mathrm{C}$ for $5 \mathrm{~min}, 40$ cycles of $95^{\circ} \mathrm{C}$ for $15 \mathrm{~s}, 56^{\circ} \mathrm{C}$ for $30 \mathrm{~s}$. The data was normalized to GAPDH and the relative transcript levels were determined using $2^{-\Delta} \mathrm{Ct}$ formula. Primers used for the RTqPCR are listed below.

Nanog-RT-f: 5'-GAAATACCTCAGCCTCCAGC-3', Nanog-RT-r: 5'-GCGTCACACCATTGCTATTC-3', Oct4-RT-f: 5'-AGAACATGTGTAAGCTGCGG-3', Oct4-RT-r: 5'-GTTGCCTCTCACTCGGTTC-3', Nestin-RT-f: 5'-GGTCTCTTTTCTCTTCCGTCC-3', Nestin-RT-r: 5'-CTCCCACATCTGAAACGACTC-3', Pax6-RT-f: 5'-GCCCTCACAAACACCTACAG-3', Pax6-RT-r: 5'-TCATAACTCCGCCCATTCAC-3', Sox1-RT-f: 5'-CAGCAGTGTCGCTCCAATCA-3', Sox1-RT-r: 5'-GCCAAGCACCGAATTCACAG-3'.

Spontaneous differentiation of NPCs was performed by maintaining NPCs on matrigel-coated plates in DMEM supplemented with $10 \%$ FBS for a week.

Intracranial tumor formation. Animal research experiments were conducted under the regulations of the UCSD Animal Care Program, protocol number S00192M. Wildtype and edited smNPCs were dissociated using accutase (Innovative Cell Technologies), washed with PBS, and resuspended at $1 \times 10^{6}$ cells in $2 \mu \mathrm{L}$ PBS supplemented with $0.1 \%$ BSA per animal. Resuspended cells were kept on ice and were inoculated into the striatum of 4-6 week-old female Nod/Scid mice (Charles River Laboratory) by stereotactic injections (1.0 mm anterior and 2.0 
$\mathrm{mm}$ right to the bregma, and $3 \mathrm{~mm}$ deep from the inner plate of the skull). Wildtype hiPSCs were injected as a control as well.

Immunohistochemistry. Paraffin-embedded tissue blocks were sectioned using the UCSD Moore's Cancer Center Pathology Core and the Center for Advanced Laboratory Medicine (UCSD). Paraffin-embedded tissue sections underwent immunohistochemical analysis using primary antibodies to GFAP (Dako), Olig2 (Sigma), Ki-67 (Abcam), and human nuclear antigen (Abcam). Immunohistochemical stains for GFAP and Olig2 were graded as follows: 0 (no cells stained $), 1+(<5 \%$ tumor cells stained $), 2+(5-50 \%$ cells stained $)$, and $3+(>50 \%$ cells stained). Ki-67 positivity was manually counted on 6 different high power fields for each model.

Sphere cell culture of iHGG. Tumors were excised from mouse brains and cut in small pieces using a scalpel, and then incubated in $3.6 \mathrm{ml}$ of Hank's Balanced Salt Solution (HBSS, Sigma) with $0.4 \mathrm{ml}$ of $10 \mathrm{x}$ Trypsin solution (Sigma) at $37^{\circ} \mathrm{C}$ for $20 \mathrm{~min}$. After incubation, $200 \mu \mathrm{l}$ of 10 $\mathrm{mg} / \mathrm{ml}$ DNaseI stock solution (Sigma) was added and incubated for 60 seconds, and then $6 \mathrm{ml}$ of HBSS was added to neutralize Trypsin and DNaseI. Tumor tissue was resuspended by pipetting up and down several times through a glass Pasteur pipette. Dissociated tissue was filtered through a strainer and was spun down by centrifugation at $400 \mathrm{G}$ for $3 \mathrm{~min}$. Cells were resuspended in $1 \mathrm{ml}$ of PBS and $9 \mathrm{ml}$ of ACK lysing buffer (Invitrogen) and were incubated at $37^{\circ} \mathrm{C}$ for $10 \mathrm{~min}$ to remove red blood cells. Approximately $1 \times 10^{6}$ cells were resuspended in 100 $\mu 1$ of MACS/BSA buffer (Miltenyi Biotec) and were incubated with $2 \mu 1$ of Fc blocking solution (BioLegend) for 5 min on ice. After blocking, $5 \mu$ l of PE-conjugated anti-human HLA-A,B,C antibody (BioLegend) was added and cells were incubated for $15 \mathrm{~min}$ on ice. Stained cells were 
washed twice with 500 $\mu 1$ of MACS/BSA buffer. PE-positive cells were then sorted using a flow cytometer (SH800, SONY). Sorted human iHGG cells were maintained in DMEM/F12 with GlutaMAX (Thermo Fisher Scientific) with 1 x B-27 supplement (Thermo Fisher Scientific), 20 ng/mL EGF (Stemcell Technologies) and 20 ng/mL bFGF (Stemcell Technologies).

Extreme Limiting dilution assay. Extreme limiting dilution assay was performed as described 22. NPCs and iHGG spheres were dissociated into single cells using accutase (Innovative Cell Technologies) and 1, 5, 10, 20, 50 and 100 cells/well were plated in 96-well plates with five replicates for each experimental condition. The total number of spheres, per well and per treatment, were quantified after 14 days in culture. Data was analyzed by extreme limiting dilution analysis (http://bioinf.wehi.edu.au/software/elda/).

Secondary tumor models and temozolomide treatment. Primary iHGG spheres were dissociated using accutase (Innovative Cell Technologies), washed with PBS, and resuspended at $2.5 \times 10^{5}$ cells in $2 \mu \mathrm{L}$ PBS supplemented with $0.1 \%$ BSA per animal. Resuspended cells were kept on ice and were inoculated as indicated above. Treatment of the mice started 7 days after inoculation of the iHGG cells by intraperitoneal injection of either vehicle (DMSO) or $50 \mathrm{mg} / \mathrm{kg}$ of temozolomide (Selleckchem). The mice were treated once daily for the first 3 days followed by 2-day drug holidays, and then once daily for 2 days again followed by 2-day drug holidays and another set of 2-day once daily treatment. This set of treatment was repeated every 4 weeks and percentage of surviving mice over time was recorded. RT-qPCR to evaluate MGMT expression was run on a CFX96 Real Time System to confirm differentiation to NPCs with the following reaction conditions: $95^{\circ} \mathrm{C}$ for $5 \mathrm{~min}, 40$ cycles of $95^{\circ} \mathrm{C}$ for $15 \mathrm{~s}, 56^{\circ} \mathrm{C}$ for $30 \mathrm{~s}$. The 
data was normalized to GAPDH and the relative transcript levels were determined using $2^{-\Delta} \mathrm{Ct}$ formula. Primers used for the RT-qPCR are listed below.

MGMT-RT-f: 5'-GCTGAATGCCTATTTCCACCA-3', MGMT-RT-r: 5'-CACAACCTTCAGCAGCTTCCA-3',

Cytogenetics. Metaphase cells were obtained by treating cells with Karyomax (Gibco) at a final concentration of $0.1 \mu \mathrm{g} / \mathrm{ml}$ for 1-3 hours. Cells were collected, washed in PBS, and resuspended in $0.075 \mathrm{M} \mathrm{KCl}$ for $15-30$ minutes. Carnoys fixative (3:1 methanol/glacial acetic acid) was added dropwise to stop the reaction. Cells were washed an additional 3 times with Carnoys fixative, before being dropped onto humidified glass slides for metaphase cell preparations. DAPI was added to the slides. Images were captured with an Olympus FV1000 confocal microscope. Spectral karyotyping analysis was performed at Applied Spectral Imaging.

Genomic DNA extracted from NPCs and iHGG cells using DNeasy blood and tissue kit (Qiagen) was analyzed by digital karyotyping using Illumina HiScan system (Illumina).

To detect DNA replication, cells were labeled with EdU and detected using the Click-iT Plus EdU Alexa Fluor 594 imaging kit (Invitrogen). Briefly, cells were pulse labeled with EdU (10 $\mathrm{uM})$ for 1 hour, then allowed to progress to metaphase for 12 hours. KaryoMax $(0.1 \mathrm{ug} / \mathrm{ml})$ was added for 3 hours to arrest cells in metaphase. The cells were then collected and metaphase spreads were prepared $^{29}$. Cells in metaphase were dropped onto a glass slide, and EdU was detected by applying the Click-iT reaction cocktail directly onto the slides for 20 minutes at room temperature. Slides were then washed with 2X SSC and mounted with anti-fade mounting medium containing DAPI. Cells in metaphase were imaged using an Olympus BX43 fluorescent microscope equipped with a QIClick camera. 
RNA sequencing. Total RNA was assessed for quality using an Agilent Tapestation, and all samples had RNA Integrity Numbers above 9.0. RNA libraries were generated using llumina's TruSeq Stranded mRNA Sample Prep Kit (Illumina) following manufacturer's instructions. RNA-seq reads were aligned to the human genome (hg19) with STAR 2.4.0h (outFilterMultimapNmax 20, outFilterMismatchNmax 999, outFilterMismatchNoverLmax 0.04, outFilterIntronMotifs RemoveNoncanonicalUnannotated, outSJfilterOverhangMin 6666 , seedSearchStartLmax 20, alignSJDBoverhangMin 1) using a gene database constructed from Gencode $v 19^{42,43}$. Reads that overlap with exon coordinates were counted using HTSeqcount (-s reverse -a 0 -t exon -i gene_id -m union) $)^{44,45}$. Raw read counts were processed with DESeq2 $2^{46}$ and only genes with mean read count over 20 were considered for the analysis. Raw read counts were transformed using the variance stabilizing transformation function included in DESeq $2^{47}$. Mean and standard deviation of normalized expression were calculated for each gene and Zscores were determined by subtracting the mean from each expression value and dividing by the standard deviation.

Single cell RNA sequencing and analysis. For the single cell RNA sequencing of secondary tumor cells, the tumors were dissected from mouse brains, cut into small pieces, and then incubated in HBSS (Sigma) containing 1x trypsin (Sigma) at $37^{\circ}$ for 20 minutes, followed by mechanical dissociation using glass pipettes to obtain single cells. Cultured sphere cells were dissociated using accutase (Innovative Cell Technologies). Single cells were processed through the Chromium Single Cell Gene Expression Solution using the Chromium Single Cell 3' Gel Bead, Chip and Library Kits v2 (10X Genomics) as per the manufacturer's protocol. In brief, 
single cells were resuspended in $0.04 \%$ BSA in PBS. Ten thousand total cells were added to each channel with an average recovery of 3,040 cells. The cells were then partitioned into Gel Beads in Emulsion in the Chromium instrument, where cell lysis and barcoded reverse transcription of RNA occurred, followed by amplification, shearing and 5' adaptor and sample index attachment. Agilent High Sensitivity D5000 ScreenTape Assay (Aglient Technologies) was performed for QC of the libraries. Libraries were sequenced on an Illumina NovaSeq. Demultiplexing, alignment to the hg19 transcriptome and unique molecular identifier (UMI)collapsing were performed using the Cellranger toolkit (version 2.0.1) provided by $10 \mathrm{X}$ Genomics. A total of 42,558 cells with approximately 53,000 mapped reads per cell were processed. Analysis of output digital gene expression matrices was performed using the Scanpy v1.3.3 package $^{48}$. Matrices for all samples were concatenated and all genes that were not detected in at least 20 single cells were discarded, leaving 20,521 genes for further analyses. Cells with fewer than 600 or more than 8,000 expressed genes as well as cells with more than 80,000 transcripts or $0.1 \%$ mitochondrial expressed genes were removed from the analysis. For the different sample subset combination analysis filtering steps were the same with the exception of specific gene and transcript thresholds for which cells were removed: $P T E N^{/-} ; N F 1^{-/}$spheres (fewer than 600 or more than 7000 expressed genes and more than 50,000 transcripts), $P T E N^{-/}$ ;NF $1^{-/}$tumors (fewer than 600 or more than 8,000 expressed genes and more than 80,000 transcripts), $T P 53^{-/-} ; P D G F R A^{48-9}$ spheres (fewer than 600 or more than 7,000 expressed genes and more than 70,000 transcripts), TP53 ${ }^{-/} ; P D G F R A^{48-9}$ tumors (fewer than 600 or more than 6,000 expressed genes and more than 40,000). Data were log normalized and scaled to 10,000 transcripts per cell. Top 4,000 variable genes were identified with the filter_genes_dispersion function, flavor='cell_ranger'. PCA was carried out, and the top 25 principal components were 
retained $\left(21,20,23\right.$ and 24 for $P T E N^{--} ; N F 1^{-/}$spheres, $P T E N^{-/} ; N F 1^{-/}$tumors, $T P 53^{-/-}$ ;PDGFRA $A^{48-9}$ spheres and $T P 53^{-/} ; P D G F R A^{48-9}$ tumors, respectively). With these principal components, neighborhood graphs were computed with 20 neighbors and standard parameters with the pp.neighbors function. Louvain clusters were computed with the tl.louvain function and standard parameters (and 0.4, 0.4, 0.7 and 0.4 resolution for $P T E N^{--} ; N F 1^{-/}$spheres, $P T E N^{--}$ ;NF1 $1^{-/-}$tumors, $T P 53^{-/} ; P D G F R A^{48-9}$ spheres and $T P 53^{-/-} ; P D G F R A^{48-9}$ tumors, respectively). Single cell and mean expression per sample heatmaps were generated with the pl.heatmap and pl.matrixplot functions, respectively. Single cell scores for TCGA molecular subtypes as well as stemness and cell cycle genes (see Supplementary Table 1) were computed with the tl.score_genes and tl.score_genes_cell_cycle functions, respectively. Differentially expressed genes were determined for each set of Louvain clusters with the tl.rank_gene_groups function (method='wilcoxon'). For Gene Ontology analysis of primary and secondary spheres, differentially expressed genes of each Louvain cluster with $\log 2$ fold over 0.5 and p-adjusted values under 0.05 were used as inputs (see Supplementary Table 2) on Metascape ${ }^{49}$ (multiple gene list option and standard parameters), using all expressed genes in the 14 samples as background. Enrichment results are summarized in Supplementary Table 3.

Statistical analyses. All statistical analyses were performed using GraphPad Prism 6 software. Data are representative of results obtained in at least 3 independent experiments. Data sets were analyzed by unpaired t-test to determine significance $(\mathrm{p}<0.05)$. Kaplan-Meier curves and comparison of survival were analyzed using Long-rank (Mantel-Cox) test. 
a

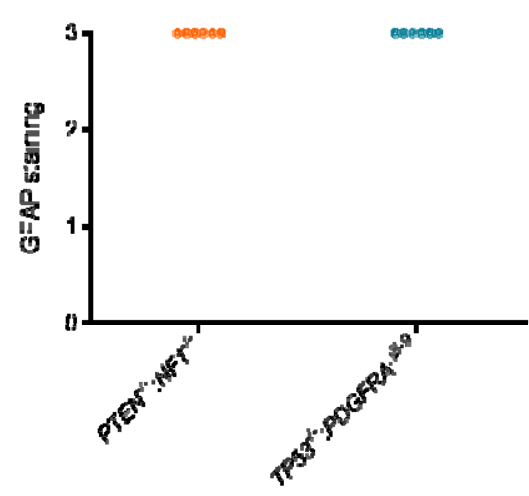

b

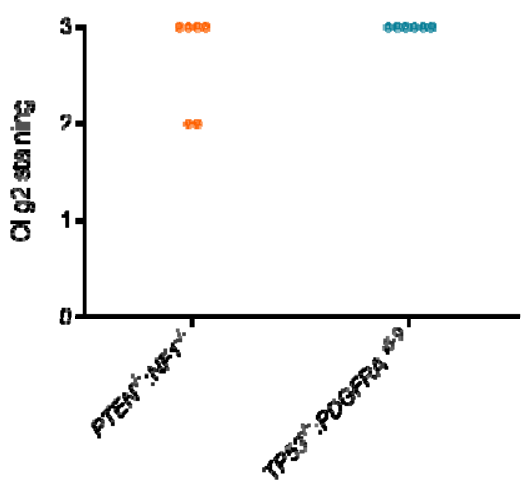

c

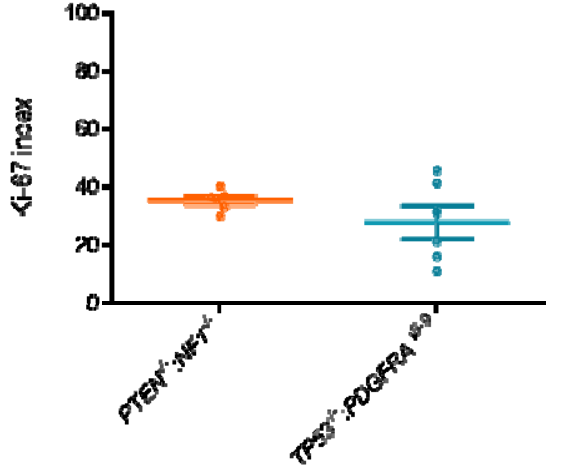

\section{Supplementary Fig. 1}

iHGG shows high GFAP, Olig2, and Ki-67 positivity. Quantification of IHC staining of

iHGGs for (a) GFAP and (b) Olig2. (c) Quantification of Ki-67 positivity. 


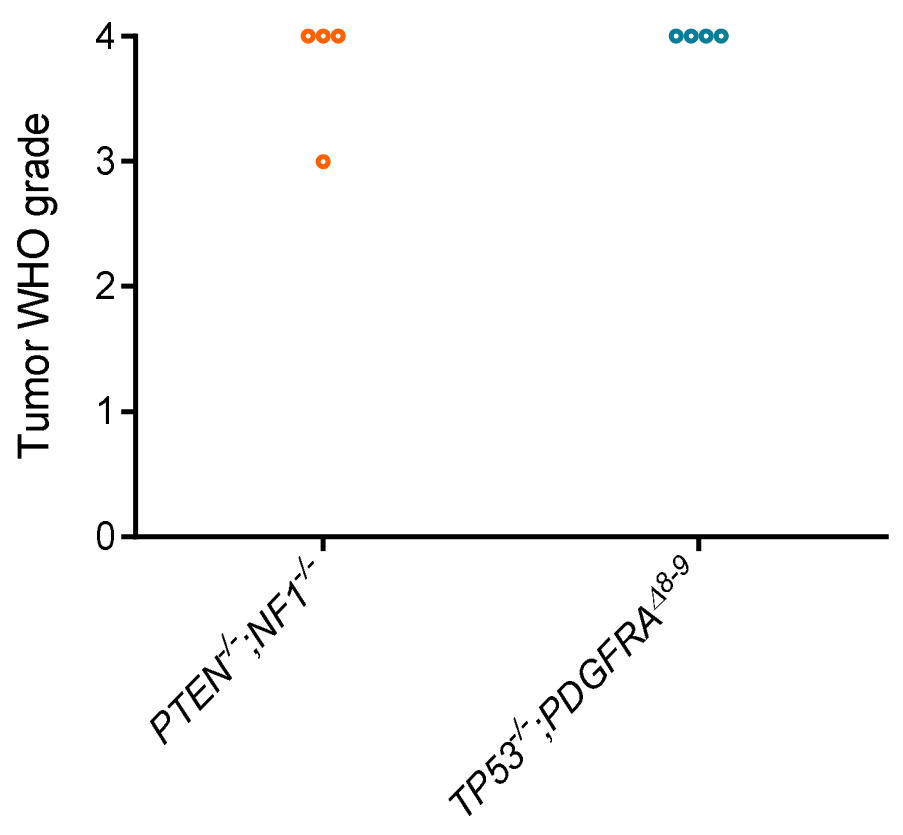

\section{Supplementary Fig. 2}

iHGG shows features of WHO grade 3 and 4 gliomas. (a-c) iHGG tumors graded as WHO

grade 3 and 4 gliomas. 

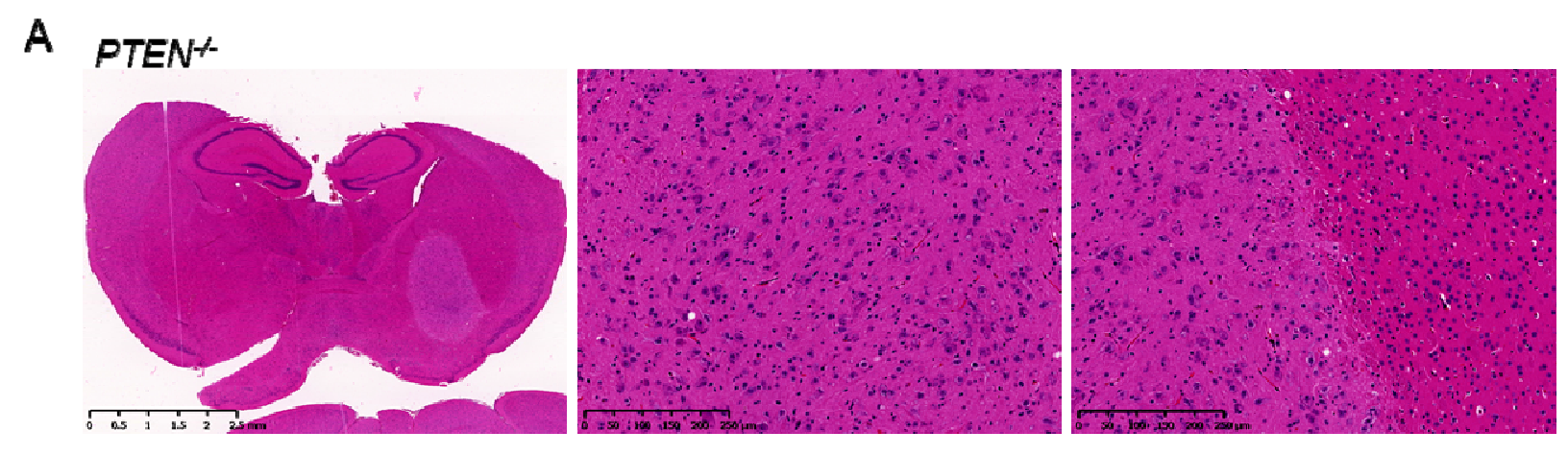

\section{B}
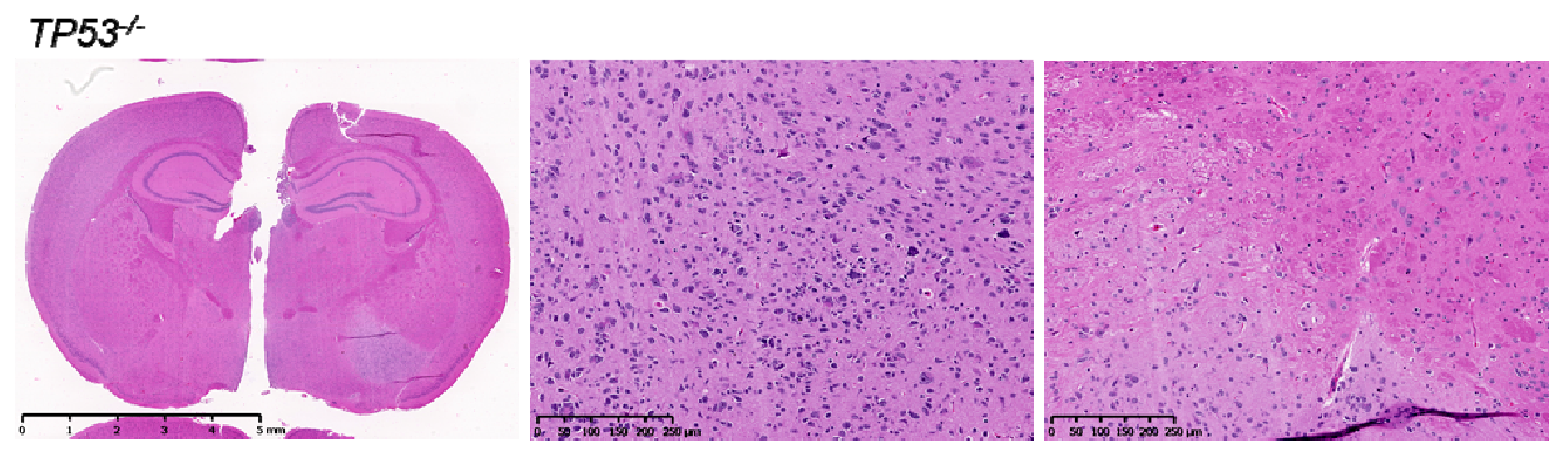

\section{Supplementary Fig. 3}

$P T E N^{/-}$and TP53 $^{-/}$singly edited NPCs do not give rise to tumors. (A) Hematoxylin and eosin staining of mouse brain 9 months after injection of $P T E N^{--}$NPCs. (B) Hematoxylin and eosin staining of the brain 9 months after injection of $T P 53^{-/} \mathrm{NPCs}$. 
A

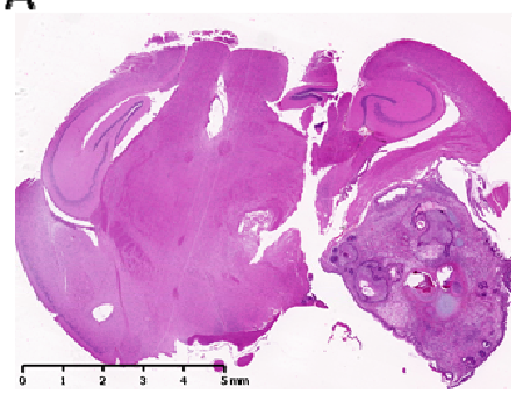

D

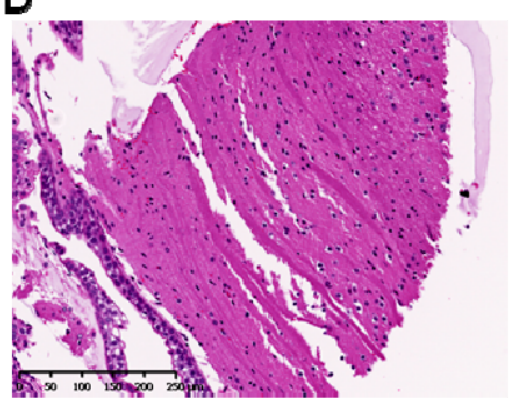

B

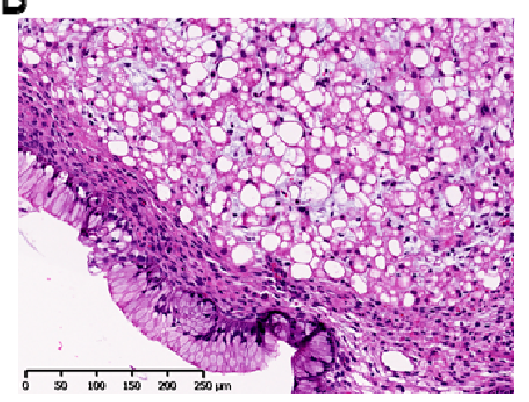

E

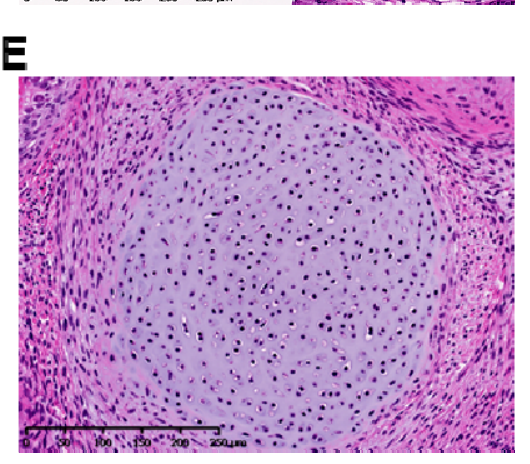

C
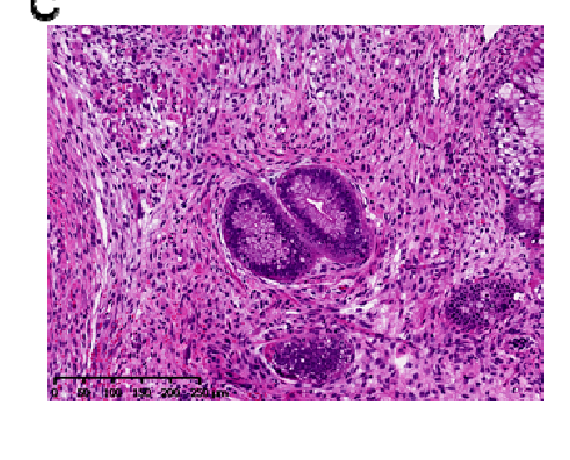

\section{Supplementary Fig. 4}

Wildtype hiPSCs form teratoma-like tumors in the mouse brain. (A) Hematoxylin and eosin staining of mouse brain post-injection of wildtype hiPSCs. (B-E) Hematoxylin and eosin staining of tumors. (B) Adipose tissue-like, (C) gastrointestinal tract-like, (D) muscle-like, and (E) cartilage-like tissues are observed. 


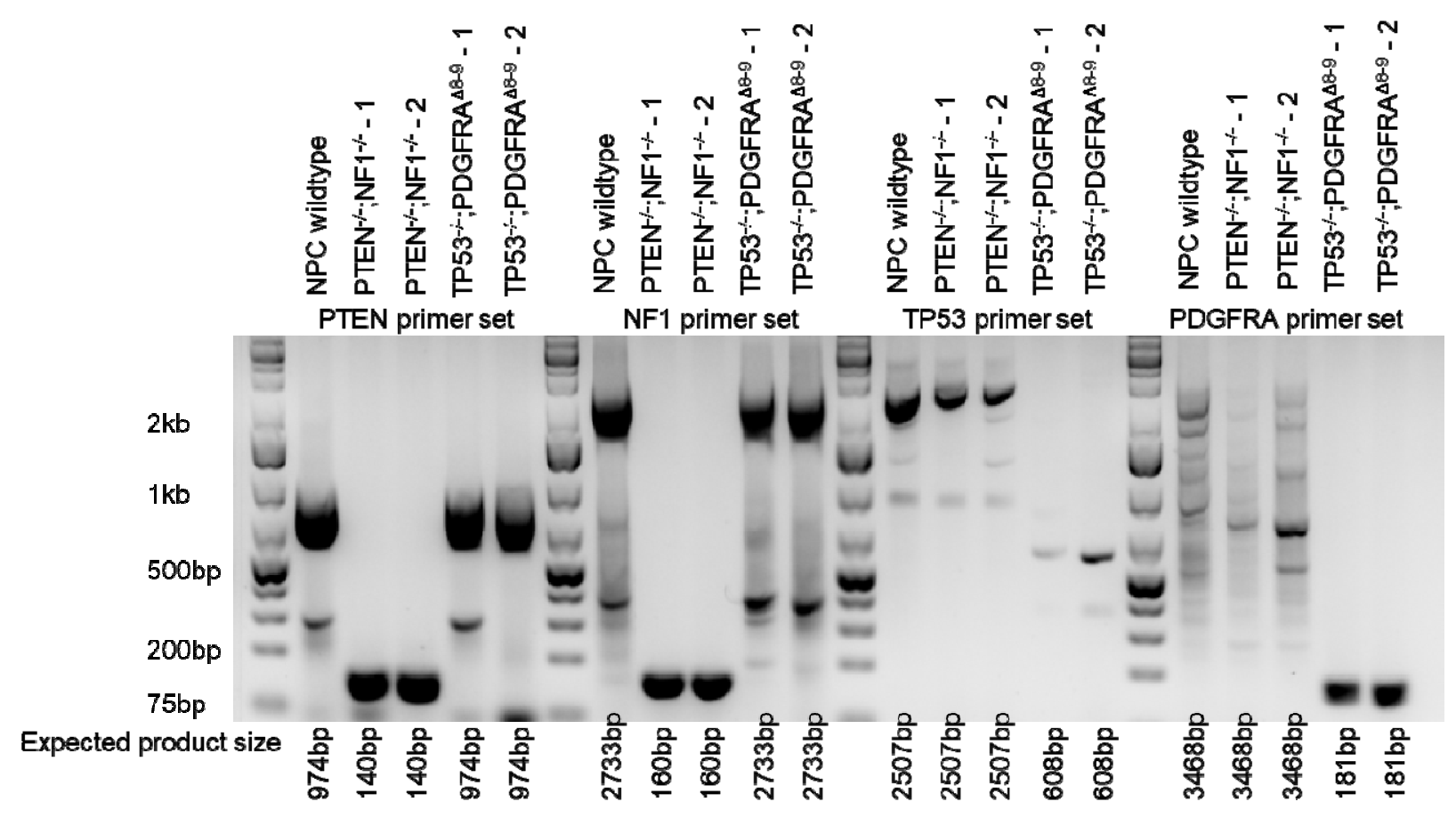

\section{Supplementary Fig. 5}

iHGG samples have the same genotype as pre-engraftment NPCs. Agarose gel

electrophoresis of amplicons from genotyping PCR for each gene in iHGG samples. PCR

amplicons from targeted alleles were expected to be 140,160, 608, and 181 bases for PTEN, NF1, TP53, and PDGFRA, respectively. 


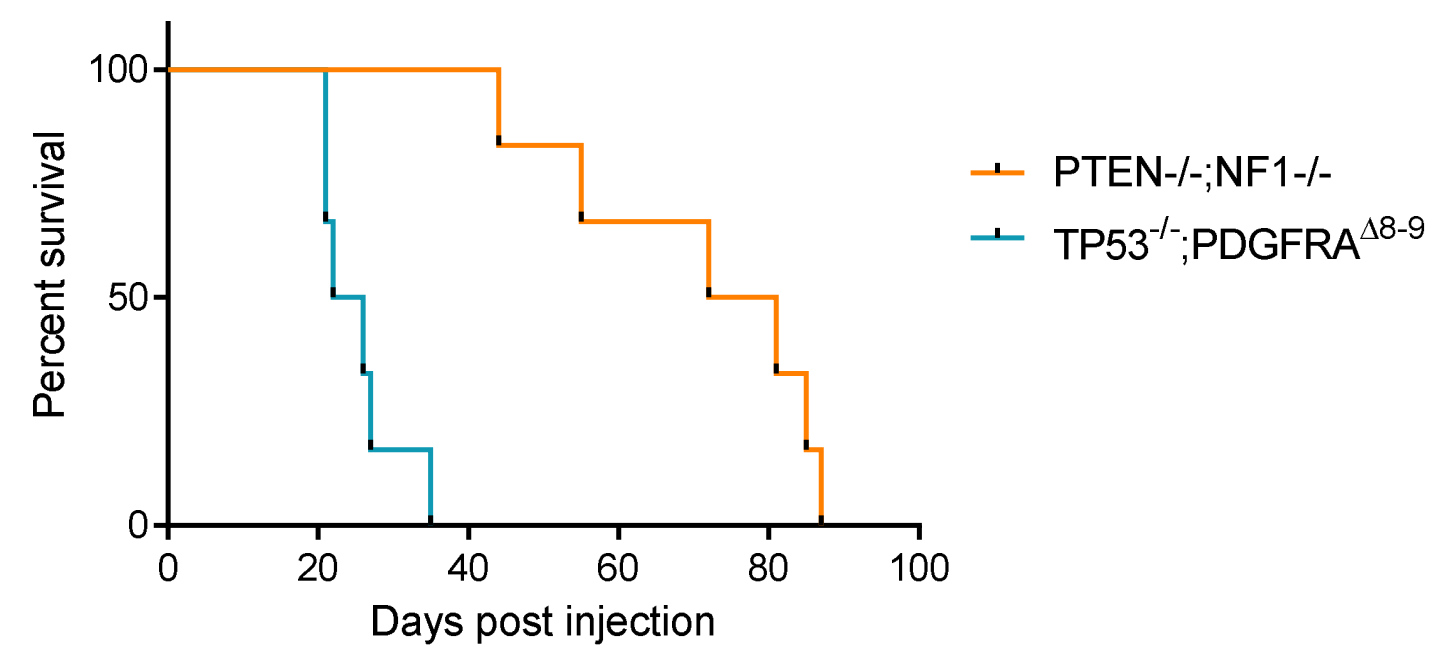

\section{Supplementary Fig. 6}

Survival of mice with the secondary iHGGs. Kaplan-Meier curves of mice engrafted with the primary iHGGs. Vehicle treated groups from the experiments shown in Fig. 3. 


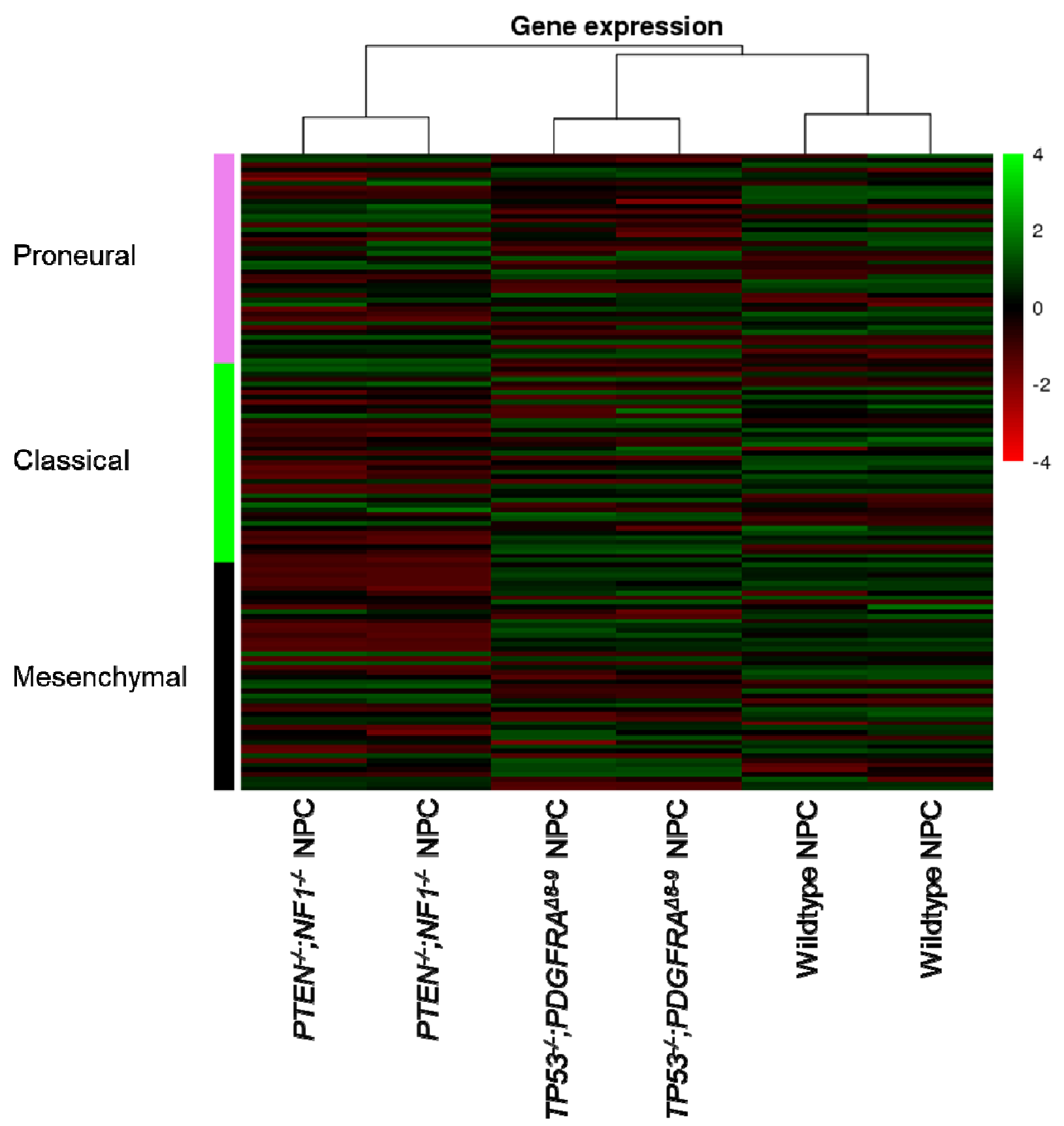

\section{Supplementary Fig. 7}

NPCs with different genetic modifications lack GBM subtype specific signatures that are observed in each corresponding iHGG. Heatmap for GBM subtype gene transcript levels of wildtype, $T P 53^{-/} ; P D G F R A^{48-9}$, and $P T E N^{-/} ; N F 1^{-/}$NPCs. 


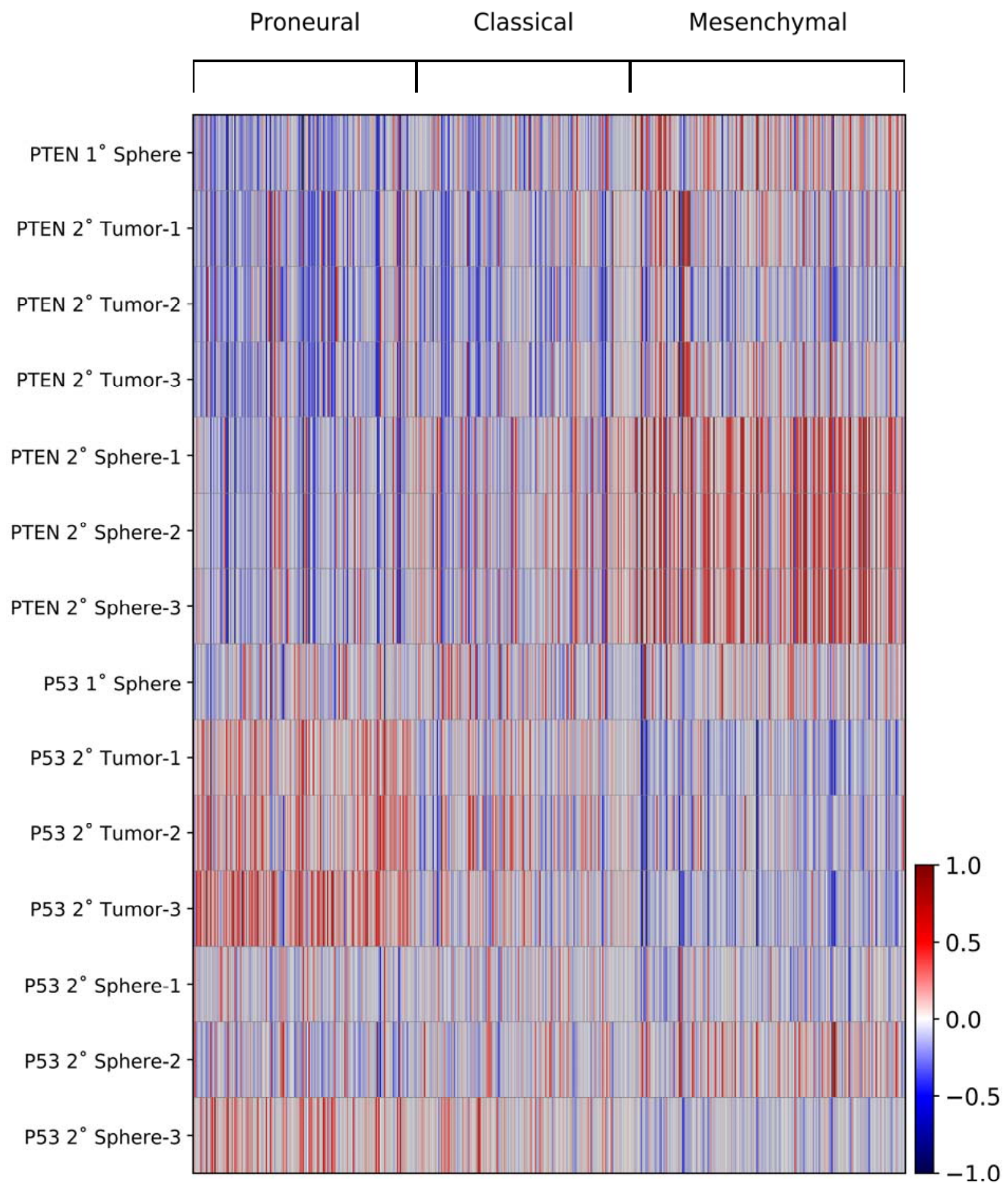

\section{Supplementary Fig. 8.}


iHGG models show inter-sample heterogeneity with characteristic molecular subtype signatures. Heatmaps of average gene expression, per sample, for each TCGA molecular subtype classifier gene. 
a
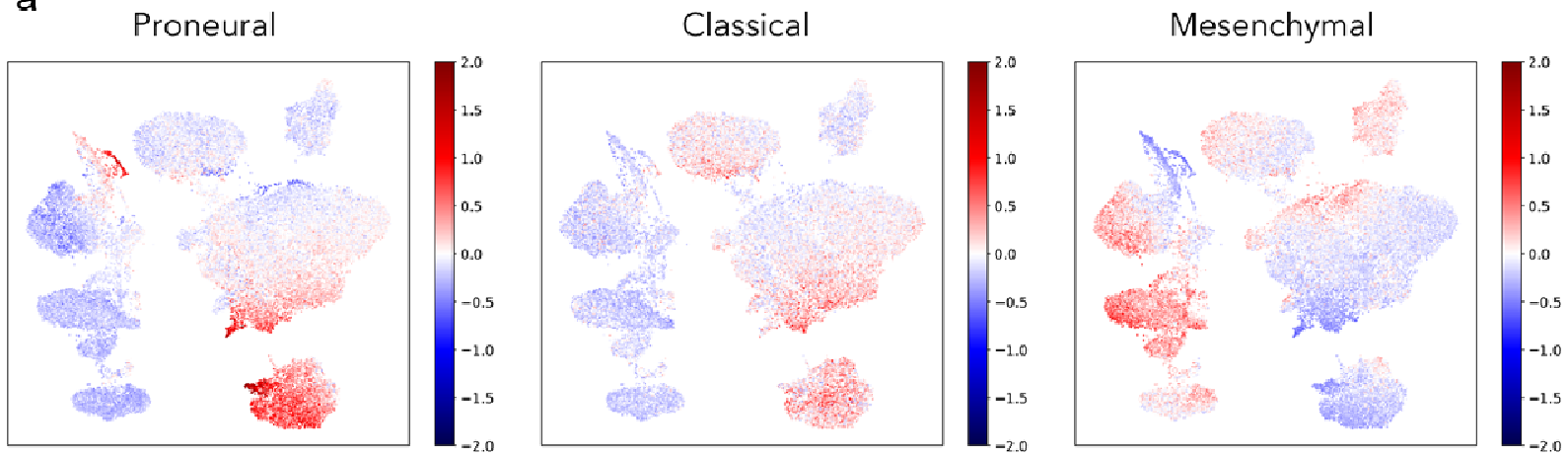

$\mathrm{b}$
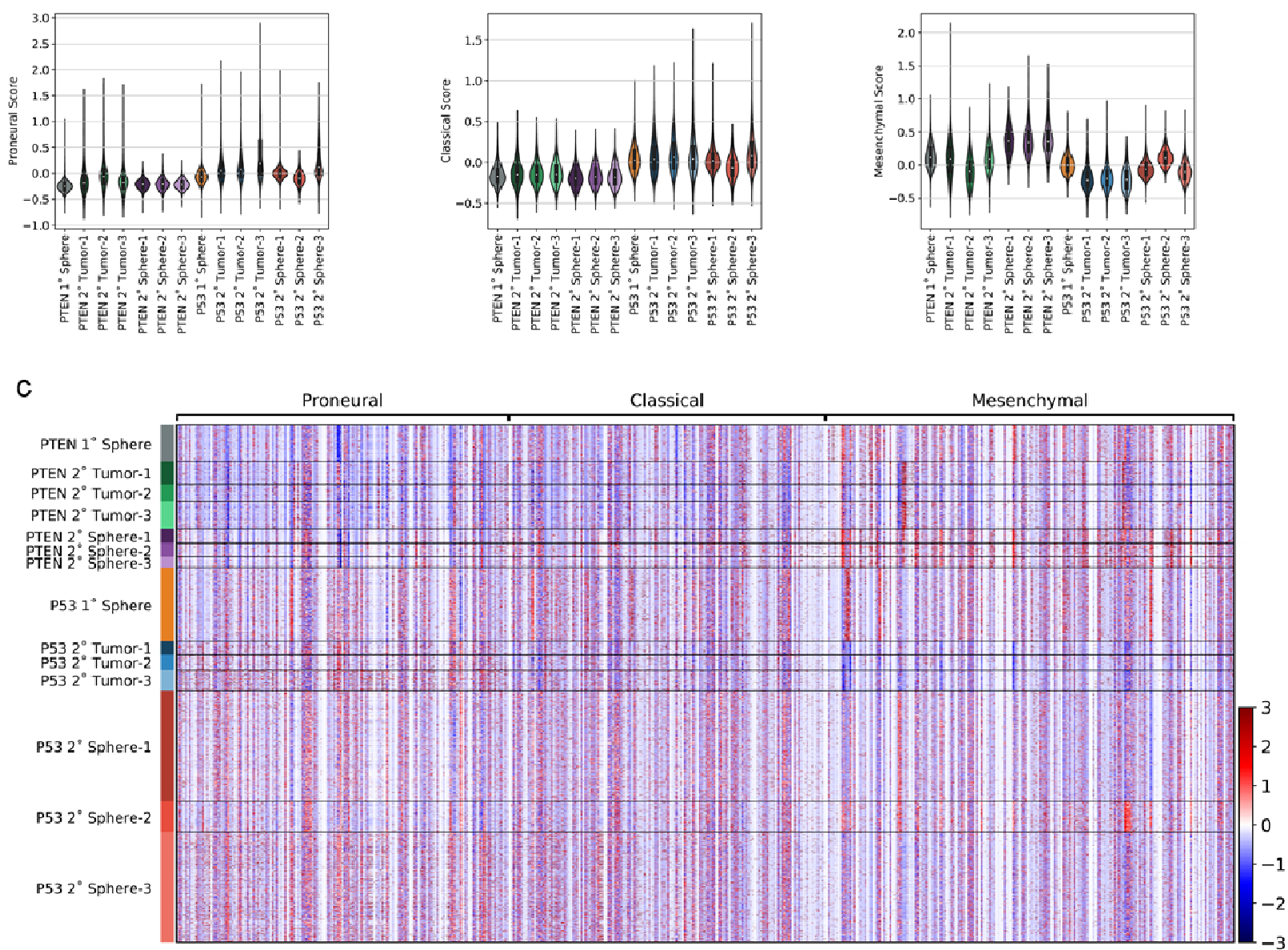

Supplementary Fig. 9

iHGG samples present intra-tumor heterogeneity with subpopulations of different GBM

molecular subtypes. Subtype scores were calculated for each cell and results overlaid on a 
bioRxiv preprint doi: https://doi.org/10.1101/576009; this version posted October 25,2019 . The copyright holder for this preprint (which was not certified by peer review) is the author/funder. All rights reserved. No reuse allowed without permission.

UMAP plot (a) or summarized as violin plots (b). Heatmaps (c) of gene expression, for each cell in all samples, for each TCGA molecular subtype classifier gene. 
a Grouped by Cell Cycle Phase

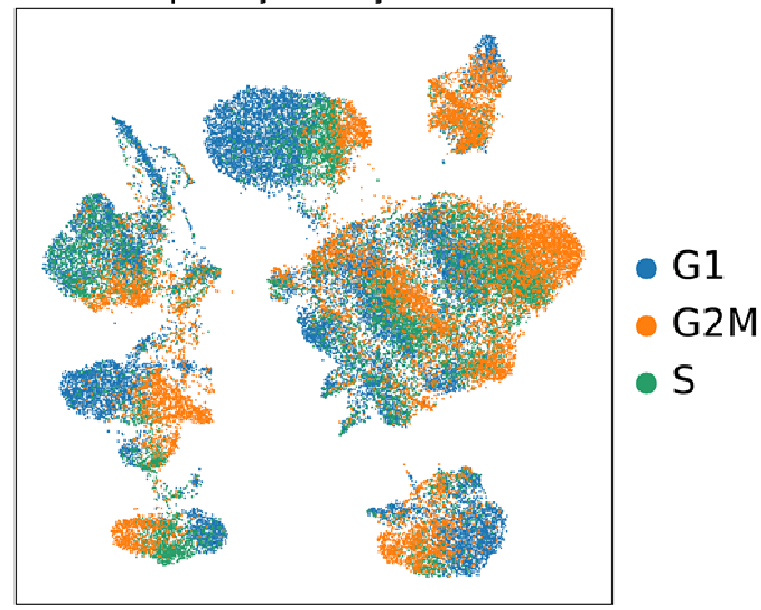

C

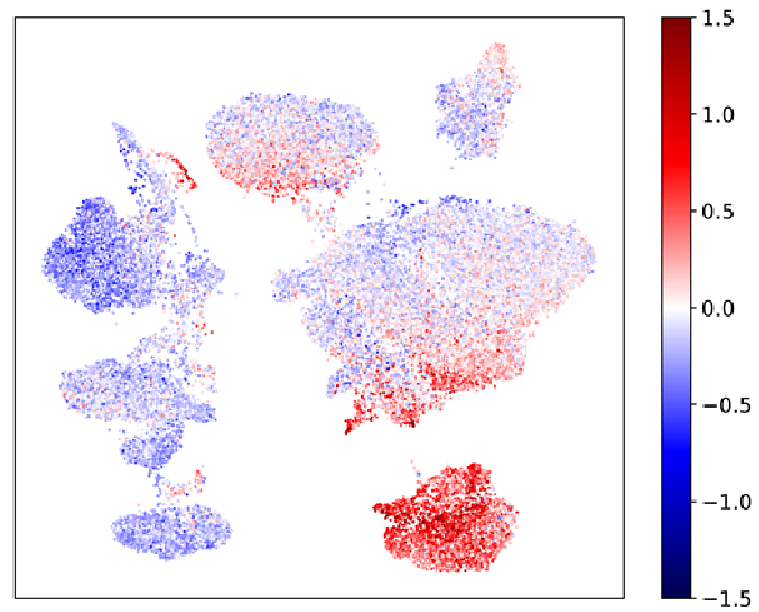

b

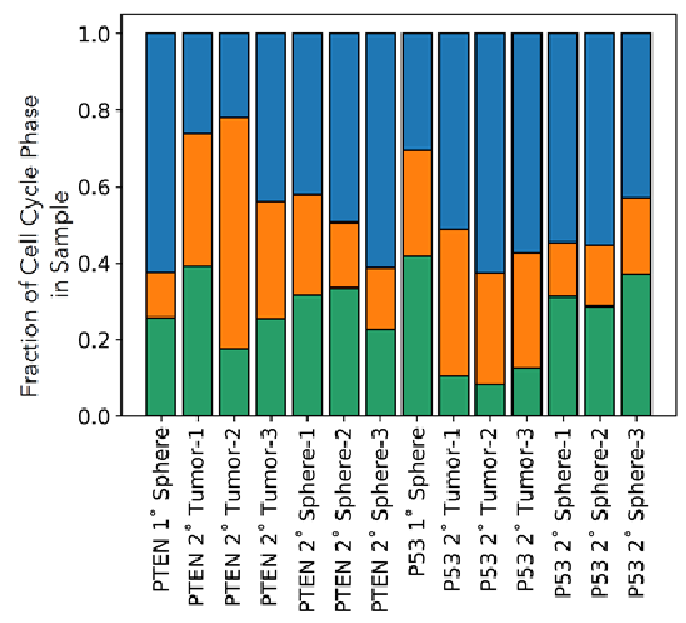

d

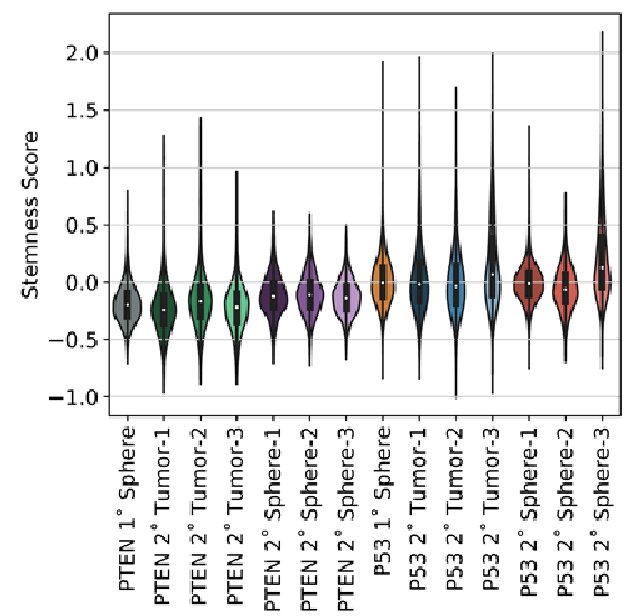

e

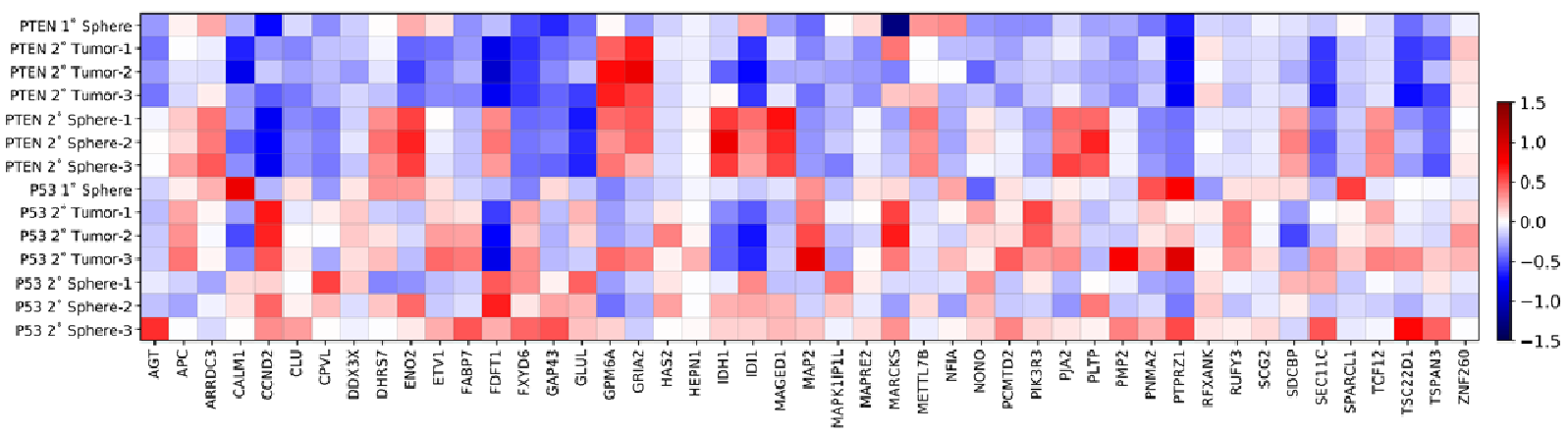

\section{Supplementary Fig. 10}


Stemness and cell cycle signatures of iHGG models. Cells were categorized based on their cell cycle status (G1, G2M or S), overlaid on a UMAP plot (a) and their distribution was calculated for each sample (b). Stemness scores were calculated for each cell and results overlaid on a UMAP plot (c) or summarized as violin plots for each sample (d). (e) Heatmap of stemnessrelated genes based on average gene expression of individual cells in each sample. 

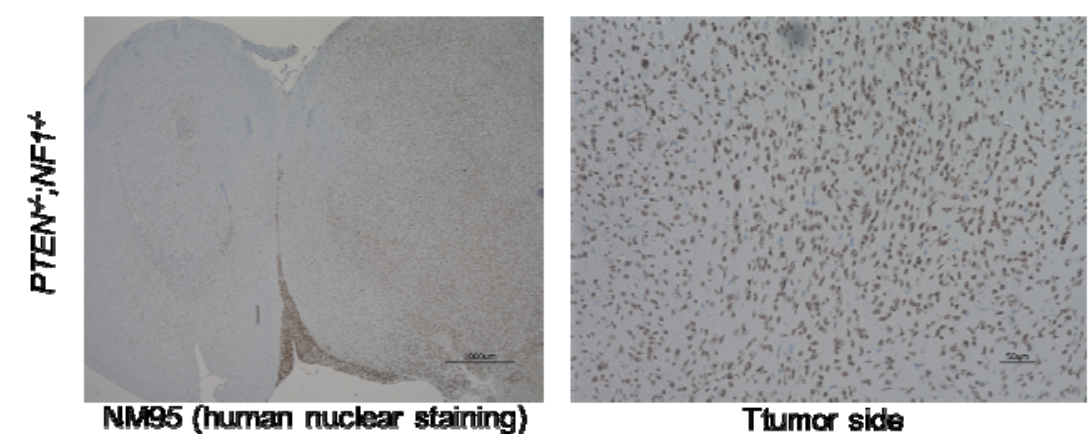

Thumor side

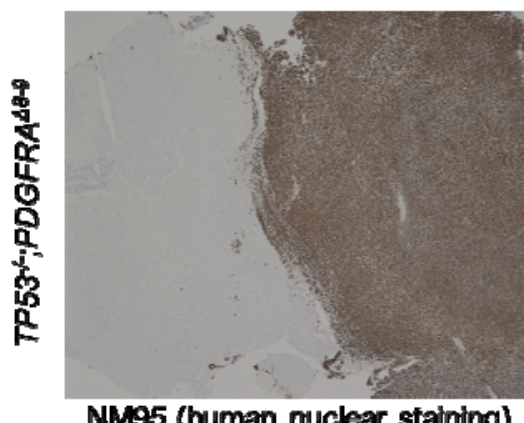

NM95 (human nuclear staining)

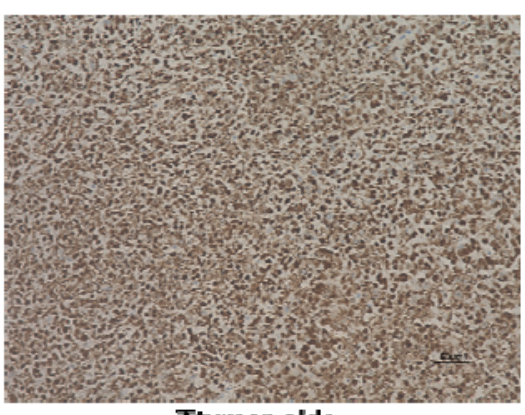

Ttumor side

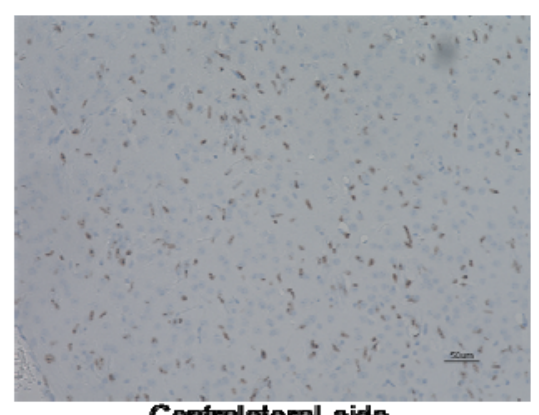

Contralateral side

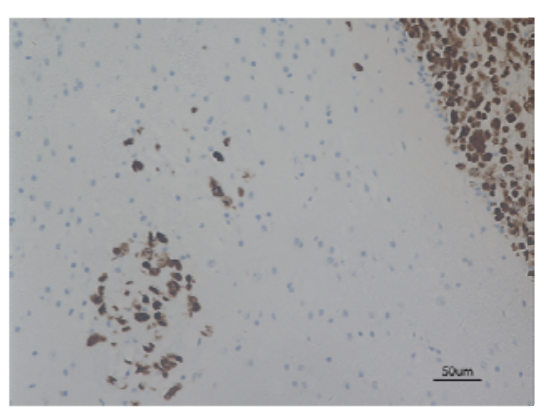

Contralateral side

\section{Supplementary Fig. 11}

PTEN $^{-/} ; \mathrm{NFI}^{-/}$and $\mathrm{TP53}^{--} ;$PDGFRA $^{48-9}$ iHGGs possess different invasive phenotypes.

NM95 human nuclear antibody staining of mouse brains with each iHGG tumor. 

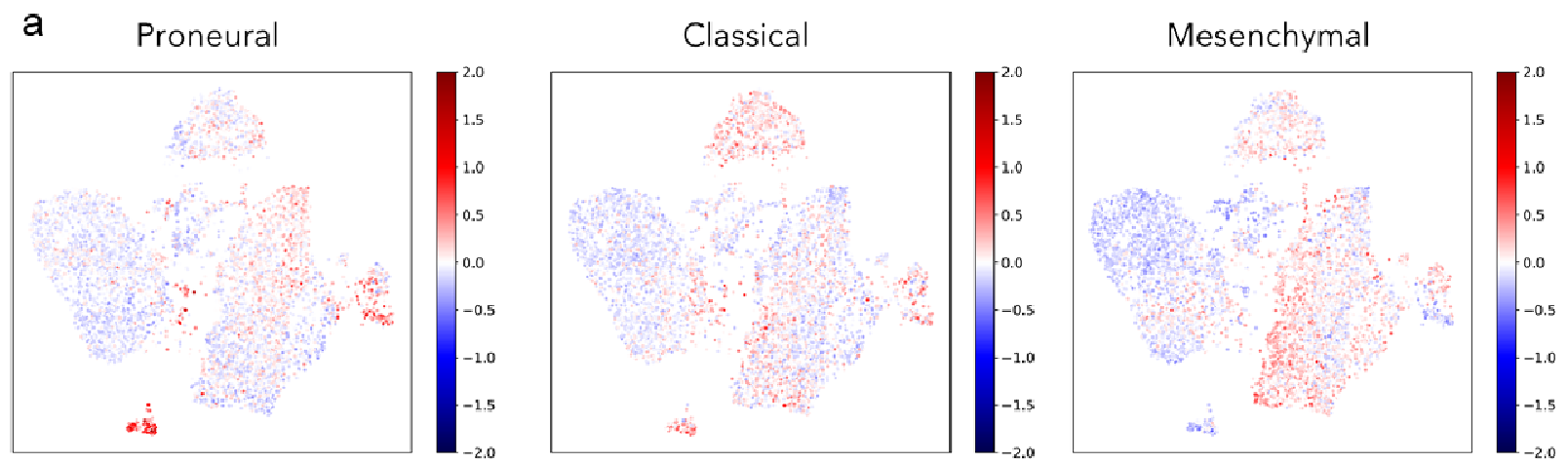

b
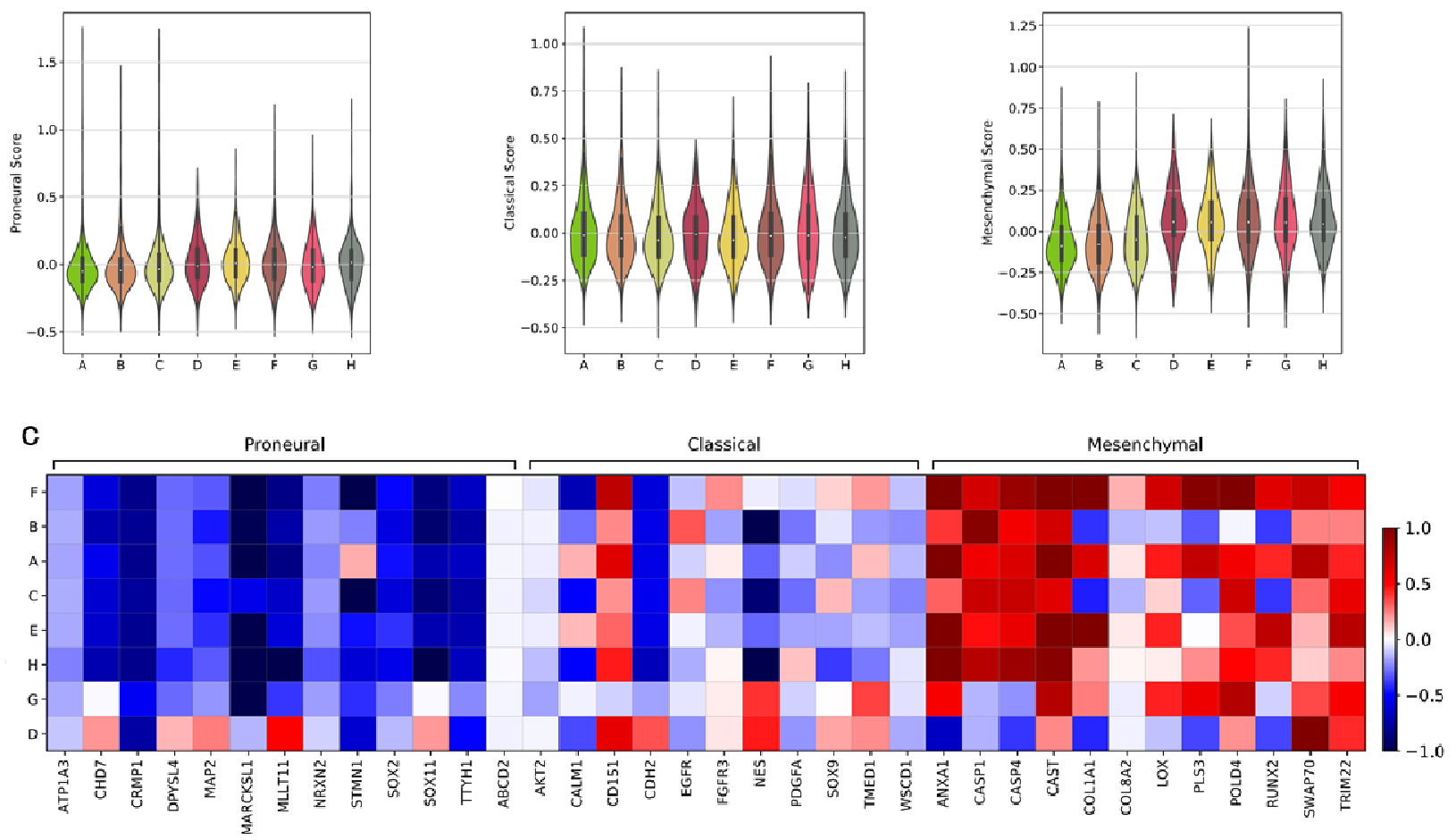

\section{Supplementary Fig. 12}

iHGG $P T E N^{-/} ; \mathrm{NF}^{-/-}$spheres present intra-tumor heterogeneity with subpopulations of different GBM molecular subtypes present for each Louvain cluster. Subtype scores were calculated for each cell and results overlaid on a UMAP plot (a) or summarized as violin plots (b). Heatmap (c) of average gene expression, for each Louvain clusters found in iHGG PTEN ${ }^{-1}$ $; N F 1^{-/}$spheres , for each TCGA molecular subtype classifier gene. Heatmap scaled values correspond to those calculated for all 14 samples. 
a

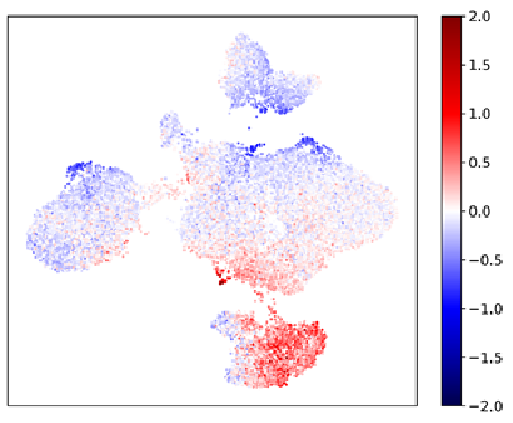

b

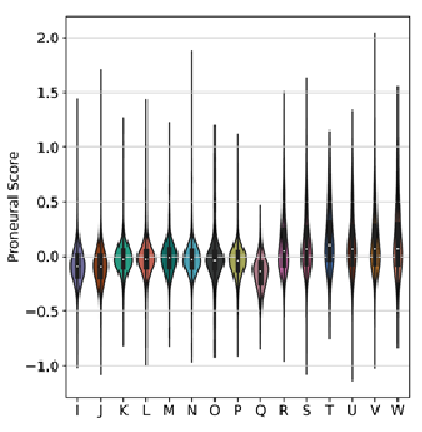

$\mathrm{C}$

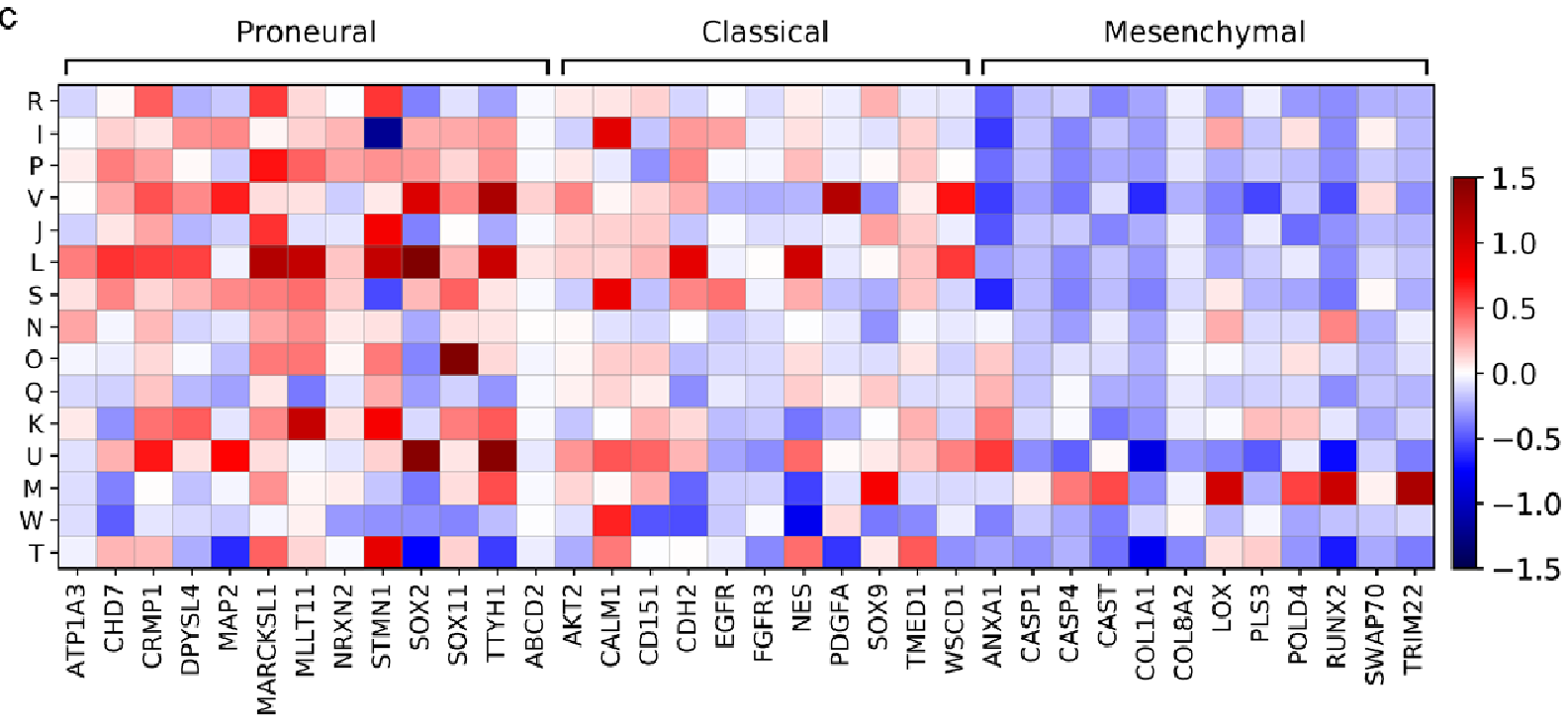

\section{Supplementary Fig. 13}

iHGG TP53 $^{-/-} ; P D G F R A^{48-9}$ spheres present intra-tumor heterogeneity with subpopulations of different GBM molecular subtypes present for each Louvain cluster. Subtype scores were calculated for each cell and results overlaid on a UMAP plot (a) or summarized as violin plots 
(b). Heatmap (c) of average gene expression, for each Louvain clusters found in iHGG $T P 53^{-/}$

;PDGFRA ${ }^{48-9}$ spheres, for each TCGA molecular subtype classifier gene. Heatmap scaled values correspond to those calculated for all 14 samples. 


\section{PTEN $\%$ NF1\%}
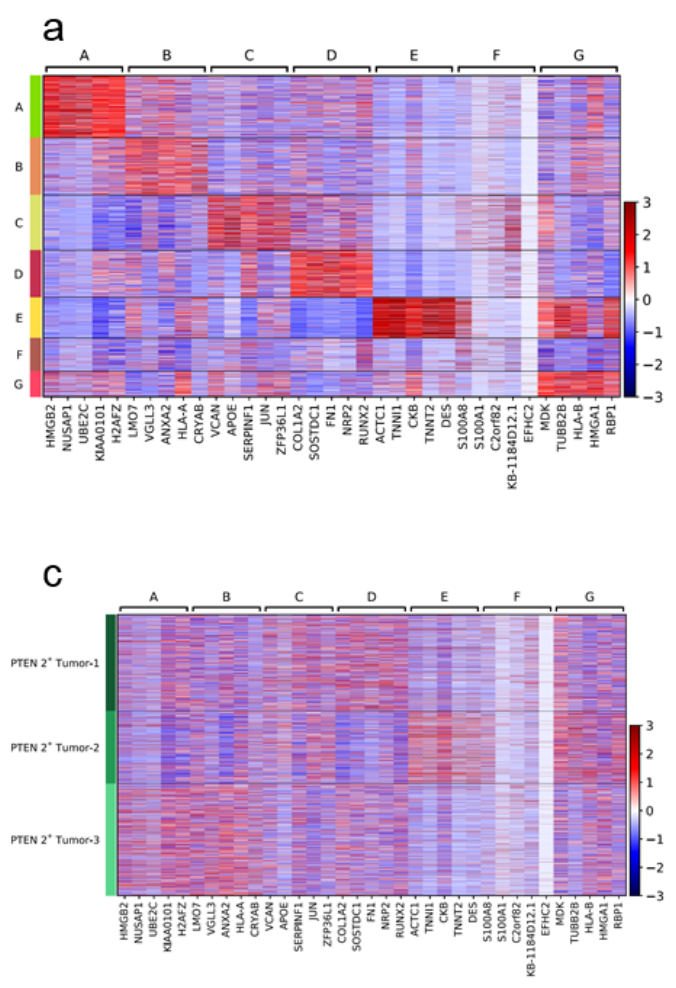

TP53/;PDGFR $\triangle 8-9$

b

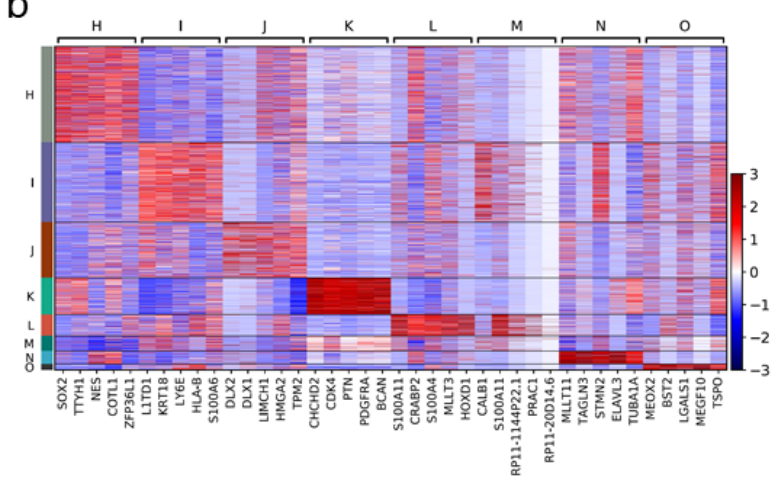

d

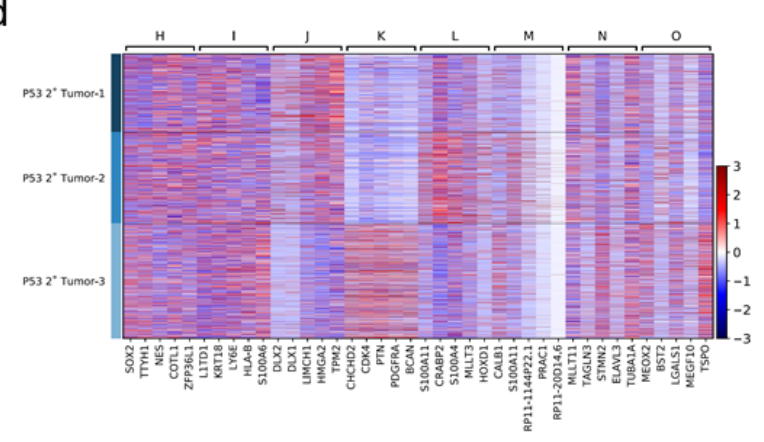

\section{Supplementary Fig. 14}

Differentially expressed genes driving molecular signatures of tumors' clusters. Heatmaps

showing all cells of top-5 Louvain cluster differentially expressed genes, plotted by cluster (a and b) or by sample identity (c and d) for $P T E N^{--} ; N F 1^{-/}$(a and c) and $T P 53^{-/-} ; P D G F R A^{48-9}$ (b and d). 